\title{
Ground States in Non-relativistic Quantum Electrodynamics
}

\author{
Marcel Griesemer ${ }^{1}$, Elliott H. Lieb² and Michael Loss ${ }^{3}$ \\ 1. Department of Mathematics, University of Alabama at Birmingham, \\ Birmingham, AL 35294 \\ 2. Departments of Physics and Mathematics, Jadwin Hall, \\ Princeton University, P. O. Box 708, Princeton, NJ 08544 \\ 3. School of Mathematics, Georgia Tech, Atlanta, GA 30332 \\ 8 July, 2000
}

\begin{abstract}
The excited states of a charged particle interacting with the quantized electromagnetic field and an external potential all decay, but such a particle should have a true ground state - one that minimizes the energy and satisfies the Schrödinger equation. We prove quite generally that this state exists for all values of the fine-structure constant and ultraviolet cutoff. We also show the same thing for a many-particle system under physically natural conditions.
\end{abstract}

\section{INTRODUCTION}

An established picture of an atom or molecule is that even in the presence of a quantized radiation field there is a ground state. The excited states that exist in the absence of coupling to the field are expected to melt into resonances, which means that they eventually decay with time into the ground state plus free photons. This picture has been established by Bach, Fröhlich and Sigal in [8] for sufficiently small values of the various parameters that define the theory. Here we show that a ground state exists for all values of the parameters (including a variable $g$-factor) in the one particle case and, under a physically appropriate assumption, in the many-particle case.

We know that the Hamiltonian for the system is bounded below, so a ground state energy always exists in the sense of being the infimum of the spectrum, but the existence of a genuine normalizable solution to the eigenvalue equation is a more delicate matter that has received a great deal of attention, especially in recent years. A physically simple example where no ground state

\footnotetext{
${ }^{1}$ Work partially supported by the Faculty Development Program of UAB

${ }^{2}$ Work partially supported by U.S. National Science Foundation grant PHY 98-20650-A01.

${ }^{3}$ Work partially supported by U.S. National Science Foundation grant DMS 00-70589.

(C) 2000 by the authors. This paper may be reproduced, in its entirety, for non-commercial purposes.
} 
exists (as far as we believe now) is the free particle (i.e., particle plus field). In the presence of an external potential, however, like the Coulomb potential of a nucleus, a ground state should exist.

The difficulty in establishing this ground state comes from the fact that the bottom of the spectrum lies in the continuum (i.e., essential spectrum), not below it, as is the case for the usual Schrödinger equation. We denote the bottom of spectrum of the free-particle Hamiltonian for $N$ particles with appropriate statistics by $E^{0}(N)$. The 'free-particle' Hamiltonian includes the interparticle interaction (e.g., the Coulomb repulsion of electrons) but it does not include the interaction with a fixed external potential, e.g., the interaction with nuclei. When the latter is included we denote the bottom of the spectrum by $E^{V}(N)$. It is not hard to see in many cases that $E^{V}(N)<E^{0}(N)$, but despite this inequality $E^{V}(N)$ is, nevertheless, the bottom of the essential spectrum. The reason is that we can always add arbitrarily many, arbitrarily 'soft' photons that add arbitrarily little energy. It is the soft photon problem that is our primary concern here.

The main point of this paper is to show how to overcome this infrared problem and to show, quite generally for a one-particle system, that a ground state exists for all values of the particle mass, the coupling to the field (fine-structure constant $\alpha=e^{2} / \hbar c$ ), the magnetic $g$-factor, and the ultraviolet cutoff $\Lambda$ of the electromagnetic field frequencies, provided a bound state exists when the field is turned off. We also include a large class of interactions much more general than the usual Coulomb interaction.

Various restricted versions of the problem have been attacked successfully. The first rigorous result, to our knowledge, is due to Arai and concerns one particle confined by an $x^{2}$-potential, the interaction with the photons being subject to the dipole-approximation. For this model, which is explicitly solvable, Arai proved existence and uniqueness of the ground state [2]. Later, Spohn showed by perturbation theory that this result extends to perturbed $x^{2}$-potentials [21]. No bounds on the parameters were needed to obtain these results but the methods oviously do not admit extensions to more realistic models.

Bach , Fröhlich and Sigal [6, 7] initiated the study of the full nonrelativistic QED model (the same model as considered in the present paper) under various simplifying assumptions. In [8] the existence of a ground state in this model for particles subject to an external binding potential was proved for $\alpha \Lambda$ small enough. The main achievement of this paper is the elimination of the earlier simplifying assumptions, especially the infrared regularization. This is the first, and up to now the only paper where a 'first principles' QED model was successfully analyzed, but with a restricted parameter range. A weaker result for the same system but with simplifications such as infrared regularization were independently obtained by Hiroshima by entirely different methods; he also showed uniqueness of the ground state, assuming its existence 14.

In a parallel development Arai and Hirokawa [3, [4, Spohn [23], and Gerard [12] investigated the ground-state problem for systems similar to the one of Bach et al in various degrees of mathematical generality. Arai and Hirokawa proved existence in what they called a 'generalized spin-boson model'. If specialized to the case of a non-relativistic $N$-particle system interacting with bosons, their result proves the existence of a ground state for confined particles and small $\alpha$. This result was extended 
in a recent preprint [4] to account for non-confined particles and systems with the true infrared singularity of QED. Concrete results in the infrared-singular case concern only special models, however, such as the Wigner-Weisskopf Hamiltonian which describes a two-level atom. Hirokawa continued this work in a recent preprint [13]. Spohn and Gerard both proved existence of a ground state for a confined particle and arbitrary coupling constant, the result of Gerard being somewhat more general [23, 12].

For the existence of a ground state in the case of massive bosons, which is a typical intermediate result in the cited works, a short and elegant proof was given by Derezinski and Gerard [10, 12] for the case of linear coupling, in which the $A^{2}$ term is omitted, and a confining potential. Some of their ideas are used in our paper.

The Hamiltonian for $N$-particles has four parts which are described precisely in the next section,

$$
H^{V}=T+V+I+H_{f}
$$

The dependence of $H^{V}$ on $N$ is not noted explicitly. The first term, $T=\sum_{j=1}^{N} T_{j}$, is the kinetic energy with 'minimal coupling' in the Coulomb gauge (i.e., $p$ is replaced by $p+\sqrt{\alpha} A$, where $A$ is the magnetic vector potential satisfying $\operatorname{div} A=0), V(X)$, with $X=\left(x_{1}, x_{2}, \ldots, x_{N}\right)$ is the external potential, typically a Coulomb attraction to one or more nuclei. In any case, we assume that $V$ is a sum of one-body potentials, i.e.,

$$
V(X)=\sum_{j=1}^{N} v\left(x_{j}\right) .
$$

The particle-particle interaction, $I$, has the important feature that it is translation invariant and, of course, symmetric in the particle labels. Both $I$ and $V$ could be spin dependent, but we shall not burden the notation with this latter possibility. Typically $I$ is a Coulomb repulsion, but we do not have to assume that $I$ is merely a sum of two-body potentials. The only requirements are: (1) the negative part of $v$ vanishes at infinity; (2) the negative part of $I$ satisfies clustering, i.e., the intercluster part of $I_{-}$tends to 0 when the spacing between any two clusters tends to infinity; (3) $V_{-}+I_{-}$are dominated by the kinetic energy as in (5). Another assumption we make (in the $N$-particle case) is that there is binding, as described below.

The natural choice for the kinetic energy is the 'Pauli operator' $T=(p+\sqrt{\alpha} A(x))^{2}+\sqrt{\alpha} \sigma \cdot B$, but we can generalize this to include the case of the usual kinetic energy $T=(p+\sqrt{\alpha} A(x))^{2}$ by introducing a 'g-factor', $g \in \mathbb{R}$. Thus, we take

$$
T=(p+\sqrt{\alpha} A(x))^{2}+\frac{g}{2} \sqrt{\alpha} \sigma \cdot B
$$

Note that $T$ is a positive operator if $0 \leq g \leq 2$ and may not be otherwise. Nevertheless, the Hamiltonian is always bounded below because of the ultraviolet cutoff we shall impose on the $A$ field, which implies that $(g / 2) \sqrt{\alpha} \sigma \cdot B+H_{f}$ is always bounded below.

We believe that the 'relativistic' operator $T=|p+\sqrt{\alpha} A(x)|$, presents no real difficulty either, but we do not want to overburden this paper with a lengthy proof. In our proof we use many 
known facts about the nonrelativistic many-body problem, such as decay of eigenfunctions; such information is more difficult to obtain for $T=|p+\sqrt{\alpha} A(x)|$.

A model that is frequently discussed is the 'Pauli-Fierz' model, but it is not entirely clear how this is defined since several variants appear in 11]. One version uses $T=(p+\sqrt{\alpha} A(x))^{2}+\sqrt{\alpha} \sigma \cdot B$, which is one of the models under consideration. Another variant uses a linearized version of this operator, $T=p^{2}+2 \sqrt{\alpha} p \cdot A(x)+\sqrt{\alpha} \sigma \cdot B$ or $T=p^{2}+2 \sqrt{\alpha} p \cdot A(x)$. These variants are not gauge invariant and, therefore, depend on the choice of gauge for $A$. Our method is applicable to these linearized models in some gauges, but not in others. We omit further discussion of this point since these variants are not the most relevant ones for quantum electrodynamics.

There is one important point that as far as we know, has not been mentioned in the QED context. This is the binding condition. Our proof of the existence of a ground state uses, as input, the assumption that

$$
E^{V}(N)<E^{V}\left(N^{\prime}\right)+E^{0}\left(N-N^{\prime}\right) \quad \text { for all } N^{\prime}<N
$$

Our work can be generalized (but we shall not do so here) to the case in which the external potential is that of attractive nuclei and these nuclei are also dynamical particles. Then, of course, it is necessary to work in the center of mass system and then (3) must be replaced by the condition that $E^{V}(N)$ is less than the lowest two-cluster threshold. While this condition, or (3) in the static case, are physically necessary for the existence of a ground state, the validity of these conditions cannot be taken for granted.

We prove inequality (3) for one particle $(N=1)$ quite generally, using only the assumption that the ordinary Schrödinger operator $p^{2}+v$ has a negative energy ground state. This certainly holds for the Coulomb potential. Indeed, one could expect, on physical grounds, that there could be binding even if $p^{2}+v$ is has no negative energy bound state, because the interaction with the field increases the effective mass of the particle - and hence the binding energy. The same argument shows that when there are $N$ particles at least one them is necessarily bound, i.e., $E^{V}(N)<E^{0}(N)$. When we consider more than one particle, we are not able to show (3) for all $N^{\prime}>1$, even if $\sum p_{j}^{2}+I+V$ has a ground state.

In the Coulomb case, it is possible to show (but we shall not do so here) that condition (3) is satisfied if the nuclear charge $Z$ is large enough. The basic idea is that if breakup into two groups, one of them with $N^{\prime}$ particles close to the nucleus and the second consisting of $N-N^{\prime}$ particles far away occurs, then there will be an attractive Coulomb tail acting on the separated particles at a distance $R$ away with net attractive potential $\left(Z-N^{\prime}\right) / R$. However, to localize one of these particles within a distance $R$ of the nucleus will require a field energy localization error of the order of $C / R$, by dimensional analysis arguments. If $\left(Z-N^{\prime}\right)>C$ then the energy can be decreased by bringing one of the unbound particles close to the nucleus.

Section 2 introduces the precise definition of our problem and the main result Theorem 2.1.

In Section 3, we show how to prove that $E^{V}(1)<E^{0}(1)$. More generally, $E^{V}(N)<E^{0}(N)$ if $v \leq 0$. 
Our strategy to establish a ground state is the usual one of showing that a minimizing sequence of trial vectors for the energy actually has a weak limit that, in fact, is a minimizer. The problem here is that one can easily construct minimizing sequences that converge weakly to zero by choosing vectors with too many soft photons. To avoid this we take a special sequence.

To define this sequence we first consider an artificial model in which the photons have a mass, i.e., $\omega(k)=\sqrt{k^{2}+m^{2}}$. Here there is no soft photon problem and we show in Section 4 that this model has a ground state $\Phi_{m}$.

In Section 5 we show that as $m \rightarrow 0$ the $\Phi_{m}$ sequence is minimizing. Then in Section 6 we use the Schrödinger equation for $\Phi_{m}$ to deduce certain properties of $\Phi_{m}$ which we call infrared bounds. One of these was proved in [8] but we need one more, which is new.

With these bounds we can show in Section 7 that $\Phi_{m}$ has a strong limit as $m \rightarrow 0$, which is a minimizer for $H^{V}$.

\section{DEFINITIONS AND MAIN THEOREM}

The Hamiltonian for $\mathrm{N}$ particles interacting with the quantized radiation field and with a given external potential $V(X)$, with $X=\left(x_{1}, x_{2}, \ldots, x_{N}\right)$ and $x_{j} \in \mathbb{R}^{3}$, is

$$
H^{V}=\sum_{j=1}^{N}\left\{\left(p_{j}+\sqrt{\alpha} A\left(x_{j}\right)\right)^{2}+\frac{g}{2} \sqrt{\alpha} \sigma_{j} \cdot B\left(x_{j}\right)\right\}+V(X)+I(X)+H_{f} .
$$

The unit of energy is $M c^{2} / 2$, where $M$ is the particle mass, the unit of length is $\ell_{c}=2 \hbar / M c$, twice the Compton wavelength of the particle, and $\alpha=e^{2} / \hbar c$ is the dimensionless "fine structure constant" (=1/137 in nature). The electric charge of the particle is $e$. The unit of time is the time it takes a light wave to travel a Compton wavelength, i. e., the speed of light is $c=1$.

The operator $p=-i \nabla$, while $A$ is the (ultraviolet cutoff) magnetic vector potential (we use the Coulomb, or radiation gauge). The unit of $A^{2}(x)$ is $M c^{2} / 2 \ell_{c}$. The magnetic field is $B=\operatorname{curl} A$ and the unit of $\mathrm{B}$ is $\alpha^{3 / 2}$ times the quantity $M^{2} e^{3} c / 4 \hbar^{3}$, which is the value of $B$ for which the magnetic length $(\hbar c / e B)^{1 / 2}$ equals the Bohr radius $2 \hbar^{2} / M e^{2}$.

The reader might wonder why we use these units, which seem to be more appropriate for a relativistic theory than for the nonrelativistic theory we are considering. Why not use the Bohr radius as the unit of length, for example? Our reason is that we want to isolate the electric charge, which is the quantity that defines the interaction of matter with the electromagnetic field, in precisely one place, namely $\alpha$. 'Atomic units' have the charge built into the length, etc. and we find this difficult to disentangle.

The Hilbert space is an appropriate subspace of

$$
\mathcal{H}=\otimes^{N} L^{2}\left(\mathbb{R}^{3} ; \mathbb{C}^{2}\right) \otimes \mathcal{F},
$$

where $\mathcal{F}$ is the Fock space for the photon field. We have in mind Fermi statistics (the antisymmetric subspace of $\left.\otimes^{N} L^{2}\left(\mathbb{R}^{3} ; \mathbb{C}^{2}\right)\right)$ and the $\mathbb{C}^{2}$ is to accomodate the electron spin. We can also deal with 
"Boltzmann statistics",in which case we would set $g=0$ and use $\otimes^{N} L^{2}\left(\mathbb{R}^{3}\right)$, or bose statistics, in which case we would set $g=0$ and use the symmetric subspace of $\otimes^{N} L^{2}\left(\mathbb{R}^{3}\right)$. These generalizations are mathematically trivial and we do not discuss them further.

For our purposes we assume that for every $\varepsilon>0$ there exists a constant $a(\varepsilon)$ such that the negative part of the potentials, $V_{-}(X)$ and $I_{-}(X)$, satisfy

$$
V_{-}+I_{-} \leq \varepsilon \sum_{j=1}^{N} p_{j}^{2}+a(\varepsilon)
$$

as quadratic forms on $\mathcal{H}$.

The vector potential is

$$
A(x)=\sum_{\lambda=1,2} \int_{|k|<\Lambda} \frac{1}{\sqrt{|k|}}\left[\varepsilon_{\lambda}(k) a_{\lambda}(k)+\varepsilon_{\lambda}(-k) a_{\lambda}^{*}(-k)\right] e^{i k \cdot x} d^{3} k
$$

where the operators $a_{\lambda}, a_{\lambda}^{*}$ satisfy the usual commutation relations

$$
\left[a_{\lambda}(k), a_{\nu}^{*}(q)\right]=\delta(k-q) \delta_{\lambda, \nu}, \quad\left[a_{\lambda}(k), a_{\nu}(q)\right]=0, \quad \text { etc }
$$

and the vectors $\varepsilon_{\lambda}(k)$ are the two possible orthonormal polarization vectors perpendicular to $k$. They are chosen for convenience in (58,59).

The number $\Lambda$ is the ultraviolet cutoff on the wavenumbers $k$. Our results hold for all finite $\Lambda$. The details of the cutoff in (6) are quite unimportant, except for the requirement that rotation symmetry in $k$-space is maintained. E.g., a gaussian cutoff can be used instead of our sharp cutoff. We avoid unnecessary generalisations.

The field energy $H_{f}$, sometimes called $d \Gamma(\omega)$ is given by

$$
H_{f}=\sum_{\lambda=1,2} \int_{\mathbb{R}^{3}} \omega(k) a_{\lambda}^{*}(k) a_{\lambda}(k) d^{3} k
$$

The energy of a photon is $\omega(k)$ and the physical value of interest to us is

$$
\omega(k)=|k|,
$$

in our units. Indeed, any continuous function that is bounded below by const. $|k|$ for small $|k|$ is acceptable. In the process of proving the existence of a ground state for $H$ we will first study the unphysical 'massive photon' case, in which

$$
\omega_{m}(k)=\sqrt{k^{2}+m^{2}}
$$

for some $m>0$, called the 'photon mass'.

In the remainder of this paper, unless otherwise stated, we shall always assume that there is no restriction on $\alpha, \Lambda$ and $g$ and that $\omega(k)$ can be either as in (9) or as in (10).

By Lemma A.5 we see easily that $H^{V}$ is bounded below for all values of the parameters, including $m=0$.

Our main theorem is 
2.1. THEOREM (Existence of a ground state). Assume that the binding condition (3) and the condition (5) hold. Then there is a vector $\Phi$ in the $N$-particle Hilbert space $\mathcal{H}$ such that

$$
H^{V} \Phi=E^{V}(N) \Phi
$$

\section{UPPER BOUND}

We shall prove the binding condition (3) for one particle and, with an additional assumption, for the $N$ particle case as well. This is that if $N$-particles are present then at least one of them binds.

As we mentioned before, at least in the single particle case, that our requirement that the system without the radiation field has a bound state is somewhat unnaturally restrictive, since one expects that the radiation field enhances binding; this has been shown to be true in the 'dipole', or Kramers approximation 15. We are able to show in the one-particle case, that the photon field cannot decrease the binding energy. It is quite possible that there could be binding even when the operator $p^{2}+v$ does not have a negative energy state, but we cannot shed any light on that question.

For the one-particle case the situation is less delicate than the $N$-particle case.

3.1. THEOREM (Binding of at least one particle). Assume that the one-particle Hamiltonian $p^{2}+v(x)$ has a negative energy bound state with eigenfunction $\phi(x)$ and energy $-e_{0}$. Then,

$$
E^{V}(1) \leq E^{0}(1)-e_{0}
$$

i.e., binding continues to exist when the field is turned on.

For the $N$-particle case we make the additional assumption that $v(x) \leq 0$ for all $x$. Then,

$$
E^{V}(N) \leq E^{0}(N)-e_{0}
$$

i.e., at least one particle is bound.

Proof. It suffices to prove that $E^{V}(N) \leq E^{0}(N)+\varepsilon-e_{0}$ for all $\varepsilon>0$. There is a normalized vector $F \in \mathcal{H}$ such that $\left(F, H^{0} F\right)<E^{0}(N)+\varepsilon$. ( $F$ is antisymmetric according to the Pauli principle.) We use the notation $\langle\cdot, \cdot\rangle$ to denote the inner product in Fock space and spin space. Then we can write $\left(F, H^{0} F\right)=\int G(X) d^{3 N} X$ with

$$
\begin{aligned}
& G(X)= \\
& \sum_{j=1}^{N}\left\{\left\langle\left(-i \nabla_{j}+\sqrt{\alpha} A\left(x_{j}\right)\right) F,\left(-i \nabla_{j}+\sqrt{\alpha} A\left(x_{j}\right)\right) F\right\rangle(X)+\sqrt{\alpha}(g / 2)\left\langle F, \sigma_{j} \cdot B\left(x_{j}\right) F\right\rangle(X)\right\} \\
& +\left\langle F,\left(I+H_{f}\right) F\right\rangle(X) .
\end{aligned}
$$

As a (unnormalized) variational trial vector we take the vector $\psi=\left[\sum_{j=1}^{N} \phi\left(x_{j}\right)^{2}\right]^{1 / 2} F$. Recall that $\phi(x) \geq 0$ since $\phi$ is the ground state of $p^{2}+v$. We also recall the Schwarz inequality

$$
\left|\frac{\sum_{j=1}^{N} \phi\left(x_{j}\right) \nabla \phi\left(x_{j}\right)}{\left[\sum_{j=1}^{N} \phi\left(x_{j}\right)^{2}\right]^{1 / 2}}\right|^{2} \leq \sum_{j=1}^{N}\left|\nabla \phi\left(x_{j}\right)\right|^{2} .
$$


Using (15), integration by parts, and the fact that $\phi$ satisfies the Schrödinger equation $\left(p^{2}+\right.$ $v) \phi=-e_{0} \phi$, we easily find that

$$
\begin{aligned}
& \left(\psi,\left[H^{V}-\left(E^{0}(N)+\varepsilon-e_{0}\right)\right] \psi\right) \\
& \leq \int\left\{G(X)-\left(E^{0}(N)+\varepsilon\right)\langle F, F\rangle(X)\right\} \sum_{j=1}^{N} \phi\left(x_{j}\right)^{2} d^{3 N} X \\
& +\int \sum_{j \neq k} v\left(x_{k}\right) \phi\left(x_{j}\right)^{2}\langle F, F\rangle(X) d^{3 N} X .
\end{aligned}
$$

When $N=1$ the last term in (16) is not present so no assumption about the potential $v$ is needed. When $N>1$ we can omit the last term because it is negative by assumption.

Now, by the $\mathbb{R}^{3}$-translation invariance of $H_{0}$, for every $y \in \mathbb{R}^{3}$ there is a 'translated' vector $F_{y}$ so that $G(X) \rightarrow G(X+(y, \ldots, y))$ and $\left\langle F_{y}, F_{y}\right\rangle(X)=\langle F, F\rangle(X+(y, \ldots, y))$. (This is accomplished by the unitary operator on $\mathcal{H}$ that takes $x_{j} \rightarrow x_{j}+y$ for every $j$ and $a_{\lambda}(k) \rightarrow \exp (i k \cdot y) a_{\lambda}(k)$.) Thus, if we denote the quantity in \{\} in (16) by $W(X)$, and if we define $\psi_{y}$ by replacing $F$ by $F_{y}$ in the definition of $\psi$, we have

$$
\begin{aligned}
& \Omega(y)=\left(\psi_{y}, H^{V}-\left(E_{0}+\varepsilon-e_{0}\right) \psi_{y}\right) \\
& \leq \int W(X+(y, \ldots, y)) \sum_{j=1}^{N} \phi\left(x_{j}\right)^{2} d^{3 N} X=\int W(X) \sum_{j=1}^{N} \phi\left(x_{j}-y\right)^{2} d^{3 N} X .
\end{aligned}
$$

Note that $\int \Omega(y) d y \leq N \int W(X) d^{3 N} X$. But $\int W(X) d^{3 N} X=\left(F,\left(H^{0}-E^{0}(N)-\varepsilon\right) F\right)$ and this is strictly negative by assumption. Hence, for some $y \in \mathbb{R}^{3}$ we have that $\Omega(y)<0$ and thus $\psi_{y} \neq 0$, which proves the theorem.

Remark: [Alternative theorem]

It may be useful to note, briefly, a different proof of Theorem 3.1, for long range potentials $v(x)$, such as the attractive Coulomb potential $-Z /|x|$, which shows that the bottom of the spectrum of $H^{V}$ lies strictly below $E^{0}$. Unfortunately, this proof does not show that the difference is at least $e_{0}$. We sketch it for the one-body case. Using the notation of the proof above, the first step is to replace $F$ by $F_{R}=u\left(x_{1} / R\right) F$ where $u$ is a smooth function with support in a ball of radius 1 . One easily finds that $\left(F_{R}, H_{0} F_{R}\right) /\left(F_{R}, F_{R}\right)=E^{0}+\varepsilon+c / R^{2}$, where $c$ is a constant that depends only on $u$ and not on $\varepsilon$ and $R$. On the other hand $\left(F_{R}, V F_{R}\right) /\left(F_{R}, F_{R}\right) \leq-Z / R$, to use the Coulomb potential as an example. To complete the argument, choose $R=2 c / Z$ and then choose $\varepsilon=c / R^{2}$. What we have used here is the fact that localization 'costs' a kinetic energy $R^{-2}$, while the potential energy falls off slower than this, e.g., $R^{-1}$.

\section{GROUND STATE WITH MASSIVE PHOTONS}

As we emphasized in the introduction, not every minimizing sequence converges to the minimizer for our $m=0$ problem, i.e., with $\omega(k)=|k|$. The situation is much easier for the massive case 
(10). The Hamiltonian in this case is given by (四) and $H_{f}$ is given by (8) with (10). To emphasize the dependence on $m$ we denote this Hamiltonian and field energy by $H_{m}^{V}$ and $H_{f}(m)$, respectively. Likewise, $E^{V}(m, N)$ and $E^{0}(m, N)$ denote the mass dependent energies, as defined before.

We emphasize that the vector potential is still given by (6), but we could, if we wished, easily replace $|k|^{-1 / 2}$ in (6) by $\left(k^{2}+m^{2}\right)^{-1 / 4}$.

It will be shown in this section that $H_{m}^{V}$ has a ground state. More precisely we prove

4.1. THEOREM (Existence of ground state). Assume that for some fixed value of the ultraviolet cutoff $\Lambda$ there is binding for the Hamiltonian $H_{m}^{V}$, i.e., $E^{V}(m, N)<\Sigma^{V}(m, N)$ where $\Sigma^{V}(m, N)=\min \left\{E^{V}\left(m, N^{\prime}\right)+E^{0}\left(m, N-N^{\prime}\right):\right.$ all $\left.N^{\prime}<N\right\}$ is the 'lowest two-cluster threshold'. Then $E(m, N)$ is an eigenvalue, i.e., there exists a state $\Phi_{m}$ in $\mathcal{H}$ such that $H_{m}^{V} \Phi_{m}=E(m, N) \Phi_{m}$.

Proof. Let us first show that it suffices to prove that for any normalized sequence $\Psi^{j}, j=1,2, \ldots$, (not necessarily minimizing) tending weakly to zero

$$
\liminf _{j \rightarrow \infty}\left(\Psi^{j}, H_{m}^{V} \Psi^{j}\right)>E^{V}(m, N)
$$

To prove this let $\Phi^{j}$ be some minimizing sequence, i.e., assume that

$$
\left\|\Phi^{j}\right\|=1
$$

and that

$$
\left(\Phi^{j}, H_{m}^{V} \Phi^{j}\right) \rightarrow E^{V}(m, N)
$$

By the Banach Alaoglu Theorem we can assume that this sequence, as well as the sequence $H_{m}^{V} \Phi^{j}$ converge weakly in the sense that for any $\Psi \in \mathcal{H}$ with $\left(\Psi, H_{m}^{V} \Psi\right)<\infty$ we have that

$$
\left(\Psi, H_{m}^{V} \Phi^{j}\right) \rightarrow\left(\Psi, H_{m}^{V} \Phi_{m}\right)
$$

where $\Phi_{m}$ is the weak limit of $\Phi^{j}$. Our goal is to show that $\left(\Phi_{m}, H_{m}^{V} \Phi_{m}\right)=E^{V}(m, N)$ and that $\left\|\Phi_{m}\right\|=1$.

Write $\Phi^{j}=\Phi_{m}+\Psi^{j}$. Obviously $\Psi^{j}$ as well as $H_{m}^{V} \Psi^{j}$ go weakly to zero. Thus

$$
\begin{aligned}
0 & =\lim _{j \rightarrow \infty}\left(\Phi^{j},\left(H_{m}^{V}-E^{V}(m, N)\right) \Phi^{j}\right) \\
& =\lim _{j \rightarrow \infty}\left(\left(\Phi_{m}+\Psi^{j}\right),\left(H_{m}^{V}-E^{V}(m, N)\right)\left(\Phi_{m}+\Psi^{j}\right)\right) \\
& =\lim _{j \rightarrow \infty}\left(\Psi^{j},\left(H_{m}^{V}-E^{V}(m, N)\right) \Psi^{j}\right)+\left(\Phi_{m},\left(H_{m}^{V}-E^{V}(m, N)\right) \Phi_{m}\right)
\end{aligned}
$$

where we used that the cross terms vanish. Since $H_{m}^{V}-E^{V}(m, N) \geq 0$ this shows that $\Phi_{m}$ minimizes the energy, and, furthermore, that

$$
0 \geq \lim _{j \rightarrow \infty}\left(\Psi^{j},\left(H_{m}^{V}-E^{V}(m, N)\right) \Psi^{j}\right) \geq \delta \liminf _{j \rightarrow \infty}\left\|\Psi^{j}\right\|^{2}
$$


for some positive constant $\delta$. The second inequality is trivial if $\lim _{\inf } \rightarrow \infty\left\|\Psi^{j}\right\|^{2}=0$ and otherwise follows from our assuption (18). This proves that $\Psi^{j}$ converges strongly to zero along a subsequence, which implies that $\left\|\Phi_{m}\right\|=1$. Hence $\Phi_{m}$ is a normalized ground state. Thus, it suffices to prove (18).

The steps that lead to a proof of (18) are quite standard. The only difficulty is that one has to localize in Fock space, which we describe first. We follow [10] with some necessary modifications and some simplifications.

Recall that, when the $a_{\lambda}^{\#}$ operators are viewed in $x$-space

$$
a_{\lambda}(f): \mathcal{F} \rightarrow \mathcal{F}, a_{\lambda}^{*}(g): \mathcal{F} \rightarrow \mathcal{F}
$$

they obey the commutation relations

$$
\left[a_{\lambda}(f), a_{\lambda}^{*}(g)\right]=\int_{\mathbb{R}^{3}} \bar{f}(x) g(x) d^{3} x=:(f, g) .
$$

Consider now two smooth localization functions $j_{1}$ and $j_{2}$ that satisfy $j_{1}^{2}+j_{2}^{2}=1$ and $j_{1}$ is supported in a ball of radius $P$. The first derivatives of $j_{1}$ and $j_{2}$ are of order $1 / P$.

The operators

$$
c_{\lambda}(f)=a_{\lambda}\left(j_{1} f\right) \otimes \mathcal{I}+\mathcal{I} \otimes a_{\lambda}\left(j_{2} f\right), c_{\lambda}^{*}(g)=a_{\lambda}^{*}\left(j_{1} g\right) \otimes \mathcal{I}+\mathcal{I} \otimes a_{\lambda}^{*}\left(j_{2} g\right)
$$

act both on the space $\mathcal{F} \otimes \mathcal{F}$. Note that

$$
\left[c_{\lambda}(f), c_{\lambda}^{*}(g)\right]=(f, g) .
$$

Thus, these new creation and anihilation operators create another Fock space $\mathcal{F}^{l}$ that is a subspace of $\mathcal{F} \otimes \mathcal{F}$ and is isomorphic to the old Fock space $\mathcal{F}$. Hence, there exists a map

$$
U: \mathcal{F} \rightarrow \mathcal{F}^{l}
$$

that is an invertible isometry between Fock spaces. It is uniquely specified by the properties

$$
a^{\#}=U^{*} c^{\#} U
$$

and the vacuum in $\mathcal{F}$ is mapped to the vacuum in $\mathcal{F} \otimes \mathcal{F}$.

Let $\phi$ and $\bar{\phi}$ be smooth nonnegative functions, with $\phi^{2}+\bar{\phi}^{2}=1, \phi$ identically one on the unit ball, and vanishing outside the ball of radius 2 . Set $\phi_{R}(X)=\phi(X / R)$. It is a standard calculation to show that for any $\Psi$ with finite energy

$$
\left(\Psi, H_{m}^{V} \Psi\right)=\left(\phi_{R} \Psi, H_{m}^{V} \phi_{R} \Psi\right)+\left(\overline{\phi_{R}} \Psi, H_{m}^{V} \overline{\phi_{R}} \Psi\right)-\left(\Psi,\left(\nabla \phi_{R}\right)^{2} \Psi\right)-\left(\Psi,\left(\nabla \overline{\phi_{R}}\right)^{2} \Psi\right) .
$$

The last two terms in (28) are bounded by const./ $R^{2}$.

One goal will be to show that for any $\Psi$ with finite energy

$$
\begin{aligned}
& \left(\Psi, \phi_{R} H_{m}^{V} \phi_{R} \Psi\right)= \\
& \left(\Psi, \phi_{R} U^{*}\left\{H_{m}^{V} \otimes \mathcal{I}+\mathcal{I} \otimes H_{f}\right\} U \phi_{R} \Psi\right)+o(1) .
\end{aligned}
$$


The error term $o(1)$ vanishes as both $R$ and $P$ go to infinity and depends otherwise only on the energy of $\Psi$. Notice that the invertible map $U$ depends on the cutoff parameter $P$ as well. (29) will be proved in Lemma A.1 in the Appendix. The intuition behind the estimate (29) is that localized electrons interact only weakly with far away photons. Those photons are described solely by their own field energy.

An immediate consequence of $(29)$ is the estimate

$$
\left(\Psi, \phi_{R} H_{m}^{V} \phi_{R} \Psi\right) \geq\left(E^{V}(m, N)+m\right)\left\|\phi_{R} \Psi\right\|^{2}-m\left(\phi_{R} \Psi, U^{*} \mathcal{I} \otimes P_{2} U \phi_{R} \Psi\right)+o(1),
$$

which is obtained by noting that the field energy in the second factor can be estimated from below by

$$
H_{f} \geq m \mathcal{I}-m P_{2}
$$

where $P_{2}$ is the projection onto the vacuum of the second factor of $\mathcal{F} \otimes \mathcal{F}$.

In a further step we prove in Lemma A.3 that the sequence

$$
\left(\phi_{R} \Psi^{j}, U^{*} \mathcal{I} \otimes P_{2} U \phi_{R} \Psi^{j}\right) \rightarrow 0
$$

as $j \rightarrow \infty$.

Returning to (28), using Corollary A.2 we have that

$$
\left(\overline{\phi_{R}} \Psi, H_{m}^{V} \overline{\phi_{R}} \Psi\right) \geq \Sigma^{V}(m, N)\left\|\overline{\phi_{R}} \Psi\right\|^{2}-o(1)
$$

with $o(1)$ going to zero as $R \rightarrow \infty$. Roughly speaking $\bar{\phi}$ forces some of the particles to be far away from the origin. Any such particle configuration can be described by two clusters with no interaction between them. In particular the interaction between these clusters via the radiation field is turned off. This means that each cluster carries its own field energy. To prove this the localization in Fock space is used. Moreover the cluster that is far away from the origin does not interact with the external potential although the repulsion among its particles is still present.

To summarize, by combining (30), (32) and (33) we have proved that

$$
\liminf \left(\Psi^{j}, H_{m}^{V} \Psi^{j}\right) \geq\left(E^{V}(m, N)+\delta\right)+o(1)
$$

where

$$
\delta=\min \left\{m, \Sigma^{V}(m, N)-E^{V}(m, N)\right\}
$$

and $o(1)$ tends to zero as $R \rightarrow \infty$ and $P \rightarrow \infty$.

\section{A MINIMIZING SEQUENCE}

We consider the Hamiltonian $H_{m}^{V}$ defined in (đ) with field energy $H_{f}(m)$ defined using $\omega_{m}(k)=$ $\sqrt{k^{2}+m^{2}}$. Our main goal here is Theorem 5.3, which shows that the ground states of the $m>0$ problem form a minimizing sequence for the $m=0$ problem. 
5.1. THEOREM $\left(E^{V}(m, N)\right.$ converges to $\left.E^{V}(0, N)\right)$. As $m \rightarrow 0$,

$$
E^{V}(m, N) \rightarrow E^{V}(0, N) \quad \text { and } \quad E^{0}(m, N) \rightarrow E^{0}(0, N)
$$

Proof. First, note that $H_{m}^{V}>H_{m^{\prime}}^{V}>H_{0}^{V}$ if $m>m^{\prime}>0$, because $\omega_{m}$ has this same monotonicity property. Therefore, for any sequence of $m \rightarrow 0, E^{V}(m, N)$ is monotonically decreasing and has a sequence-independent, finite limit, which we call $E^{*}$, and we note that $E^{*} \geq E^{V}(0, N)$. To prove the opposite, namely $E^{*} \leq E^{V}(0, N)$, we shall prove that $E^{*} \leq E^{V}(0, N)+2 \varepsilon$ for every $\varepsilon>0$.

Let $\Phi \in \mathcal{H}$ be normalized and such that $\left(\Phi, H_{0}^{V} \Phi\right)<E^{V}(0, N)+\varepsilon$.

We note that $H_{m}^{V}<H_{0}^{V}+m \mathcal{N}$, where $\mathcal{N}$ is the number operator

$$
\mathcal{N}=\sum_{\lambda=1,2} \int_{|k|<\Lambda} a_{\lambda}^{*}(k) a_{\lambda}(k) d^{3} k
$$

Thus, if we use $\Phi$ as a variational function for $H_{m}^{V}$ we have $E^{V}(m, N)<E^{V}(0, N)+\varepsilon+$ $m(\Phi, \mathcal{N} \Phi)$, and our goal is accomplished provided that $\Phi$ can be chosen so that $(\Phi, \mathcal{N} \Phi)<\infty$, in addition to $\left(\Phi, H_{0}^{V} \Phi\right)<E^{V}(0, N)+\varepsilon$. If a way can be found to modify $\Phi$ to another vector $\widetilde{\Phi}$ so that $(\widetilde{\Phi}, \mathcal{N} \widetilde{\Phi})<\infty$, in addition to $\left(\widetilde{\Phi}, H_{0}^{V} \widetilde{\Phi}\right)<E^{V}(0, N)+2 \varepsilon$ the proof will be complete.

A suitable choice is $\widetilde{\Phi}=\Pi_{n} \Phi$, with $\Pi_{n}$ being the projector onto the subspace of $\mathcal{H}$, with $n$ or fewer photons, i.e., $\mathcal{H}_{n}=\otimes^{N} L^{2}\left(\mathbb{R}^{3} ; \mathbb{C}^{2}\right) \otimes \mathcal{F}_{\leq n}$, for an appropriately large $n$. It is easy to see, with the help of Lemma A.5 that $\left(\Pi_{n} \Phi,[V+I] \Pi_{n} \Phi\right) \rightarrow(\Phi,[V+I] \Phi)$, that $\left(\Pi_{n} \Phi, H_{f} \Pi_{n} \Phi\right) \rightarrow\left(\Phi, H_{f} \Phi\right)$, and $\left(\Pi_{n} \Phi, p_{j}^{2} \Pi_{n} \Phi\right) \rightarrow\left(\Phi, p_{j}^{2} \Phi\right)$ as $n \rightarrow \infty$. The following Lemma 5.2, shows that the other terms converge as well. The same proof works for $E^{0}(m, N)$.

5.2. LEMMA (Finite photon number approximation). Let $\Phi \in \mathcal{H}$ be such that $\left(\Phi, H_{f} \Phi\right)<$ $\infty$. Denote by $\Pi_{n}$ the projection in $\mathcal{H}$ onto states with photon number less than or equal to $n$, i.e., $\Pi_{n}$ is the projection onto the subspace $\mathcal{H}_{n}=\otimes^{N} L^{2}\left(\mathbb{R}^{3} ; \mathbb{C}^{2}\right) \otimes \mathcal{F}_{\leq n} \subset \mathcal{H}$. Let $\Phi_{n}=\Pi_{n} \Phi$. Then we have the following strong convergence as $n \rightarrow \infty$ (in addition to $\left.\Phi_{n} \rightarrow \Phi\right)$

$$
\begin{aligned}
& A\left(x_{j}\right) \Phi_{n} \quad \rightarrow \quad A\left(x_{j}\right) \Phi \\
& B\left(x_{j}\right) \Phi_{n} \rightarrow B\left(x_{j}\right) \Phi .
\end{aligned}
$$

Proof. Write $A=D+D^{*}$ where $D$ contains the annihilation operators and $D^{*}$ contains the creation operators. We omit $\left(x_{j}\right)$ and we omit the vector index of $A$ for simplicity. Since $\left(\Phi, H_{f} \Phi\right)<\infty$ we learn from Lemma A.4 that $D \Phi$ and $D^{*} \Phi$ are in $\mathcal{H}$. Since $\Pi_{n-1} \rightarrow \mathcal{I}$ strongly,

$$
D \Phi_{n}=\Pi_{n-1} D \Phi \rightarrow D \Phi .
$$

The same holds if $D$ is replaced by $D^{*}$ and $n-1$ by $n+1$. This proves the statement for $A$. The statement for $B$ is proved in the same way. 
The following are two corollaries of Theorem 5.1. The first is the input for Section 6. The second is important for showing that it is only necessary to state the 'binding' condition (3) once (for $m=0$ ). It is a trivial consequence of Theorem 5.1.

5.3. THEOREM (Minimizing sequence). Suppose that $m_{1}>m_{2}>\ldots>0$ is a sequence tending to zero and suppose that $\Phi_{j}$, for $j=1,2, \ldots$ is an approximate minimizer for $H_{m_{j}}^{V}$ in the sense that

$$
\delta_{j} \equiv\left(\Phi_{j}, H_{m_{j}}^{V} \Phi_{j}\right)-E^{V}\left(m_{j}, N\right) \rightarrow 0 \quad \text { as } j \rightarrow \infty
$$

Then $\Phi_{1}, \Phi_{2}, \ldots$ is a minimizing sequence for $H_{0}^{V}$.

Proof. $\quad E^{V}\left(m_{j}, N\right)+\delta_{j}=\left(\Phi_{j}, H_{m_{j}}^{V} \Phi_{j}\right)>\left(\Phi_{j}, H_{0}^{V} \Phi_{j}\right) \geq E^{V}(0, N)$.

5.4. LEMMA (Binding without mass implies uniform binding with mass). Assume (3), i.e., assume that

$$
E^{V}(0, N)<\min \left\{E^{V}\left(0, N^{\prime}\right)+E^{0}\left(0, N-N^{\prime}\right): N^{\prime}<N\right\}-2 \varepsilon .
$$

Then, for all sufficiently small $m$,

$$
E^{V}(m, N)<\min \left\{E^{V}\left(m, N^{\prime}\right)+E^{0}\left(m, N-N^{\prime}\right): N^{\prime}<N\right\}-\varepsilon .
$$

Proof. Each of the various energies converges as $m \rightarrow 0$ by Theorem 5.1 .

\section{TWO INFRARED BOUNDS}

We have seen that any sequence of (approximate) minimizers for the $H_{m}^{V}$ problem $(m>0)$ is a minimizing sequence for the $H_{0}^{V}$ problem. A crucial point was the possibility of finding an approximate minimizer for the $H_{0}^{V}$ problem that has a finite expectation value for the total photon number operator $\mathcal{N}$.

We also know by Corollary 5.4 and Theorem 4.1 that the $H_{m}^{V}$ problem has a ground state, $\Phi_{m}$, and in this section we shall prove two theorems about the soft photon behavior of $\Phi_{m}$. The first, Theorem 6.1, is about the photon number $a_{\lambda}^{*}(k) a_{\lambda}(k)$ and is based on a method of [8]. The second, Theorem 6.3, which has no antecedent we are aware of, is about the derivative of $a_{\lambda}^{*}(k) a_{\lambda}(k)$ with respect to $k$.

6.1. THEOREM (Photon number bound). Assume that there is binding, i.e., $E^{V}(m, N)<$ $\Sigma^{V}(m, N)$. Assume that $\Phi_{m}$ is a normalized ground state for the many-body Hamiltonian $H_{m}^{V}$, $m \geq 0$. Then

$$
\left(\Phi_{m}, a_{\lambda}^{*}(k) a_{\lambda}(k) \Phi_{m}\right)<\frac{P \alpha}{|k|}\left(1+g^{2}\right) \chi_{\Lambda}(k),
$$

where $P$ is a finite constant independent of $\Phi_{m}, g, \alpha$, and depends on $m$ only via the binding energy $\Sigma^{V}(m, N)-E^{V}(m, N)>0$ and of course on $\Lambda$. The function $\chi_{\Lambda}(k)$ is the characteristic function of the ball of radius $\Lambda$. 
Remark: We have proved in Corollary 5.4 that the binding energy is a uniformly positive function of $m$ for small $m$, if the binding energy at $m=0$ is not zero. Therefore, Theorem 6.1 implies that the number operator $\mathcal{N}$ in (37) is uniformly bounded for small $m$.

The proof of Theorem 6.1 will be based on the following lemma about exponential decay of

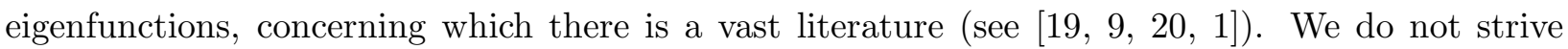
at all to get the best exponential decay constants. For us the only really relevant goal is a decay estimate that depends only on $\Sigma^{V}(m, N)-E^{V}(m, N)>0$, but not otherwise on $m$.

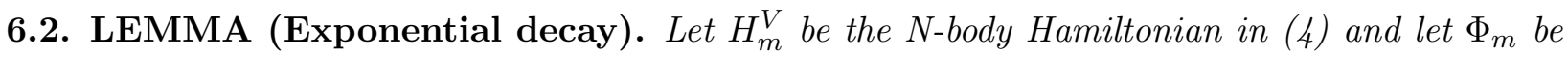
a groundstate wave function, which necessarily satisfies the Schrödinger equation

$$
H_{m}^{V} \Phi_{m}=E^{V}(m, N) \Phi_{m}
$$

We assume that $\Sigma^{V}(m, N)-E^{V}(m, N)>0$ and choose $\beta>0$ with $\beta^{2}<\Sigma^{V}(m, N)-E^{V}(m, N)$. Then

$$
\left\|\exp (\beta|X|) \Phi_{m}\right\|^{2} \leq C\left(1+\frac{1}{\Sigma^{V}(m, N)-E^{V}(m, N)-\beta^{2}}\right)\left\|\Phi_{m}\right\|^{2}
$$

where the constant $C$ does not depend on $m$.

The strategy of the following proof is probably due to Agmon [1]. We learned it from [16].

Proof. Let $G(X)$ any smooth, bounded function on $\mathbb{R}^{3 N}$ with bounded first derivative. We easily compute

$$
\left[\left[H_{m}^{V}-E^{V}(m, N), G\right], G\right]=-2|\nabla G|^{2}
$$

We use the Schrödinger equation (40) to compute

$$
\left(G \Phi_{m},\left[H_{m}^{V}-E^{V}(m, N)\right] G \Phi_{m}\right)=-\frac{1}{2}\left(\Phi_{m},\left[\left[H_{m}^{V}-E(m), G\right], G\right] \Phi_{m}\right)=\left(\Phi_{m},|\nabla G|^{2} \Phi_{m}\right) .
$$

Now we choose $G$ to be

$$
\begin{aligned}
G(X) & =\chi(X / R) \exp [f(X)], \quad \text { where } \\
f(X) & =\left[\frac{\beta|X|}{1+\varepsilon|X|}\right]
\end{aligned}
$$

and where $0 \leq \chi \leq 1$ is a smooth cutoff function that is identically equal to 1 outside the ball of radius 2 , and identically zero inside the ball of radius 1 . We let $\varepsilon \rightarrow 0$ at the end.

Next, we calculate

$$
|\nabla G|^{2}=|\nabla \chi|^{2} e^{2 f}+2 \nabla \chi \cdot \nabla f e^{f} G+|\nabla f|^{2} G^{2}
$$

and note that the first and second terms are compactly supported in $\mathbb{R}^{3 N}$ and each is bounded by a constant $C$ that depends on $\beta$ and $R$. 
Returning to (43), we obtain, after rearranging terms,

$$
\left.\left(G \Phi_{m},\left(H_{m}^{V}-E^{V}(m, N)-|\nabla f|^{2}\right)\right) G \Phi_{m}\right) \leq C\left\|\Phi_{m}\right\|^{2} .
$$

Since $|\nabla f| \leq \beta$ we know by Corollary A.2 that

$$
\begin{aligned}
& \chi\left(H_{m}^{V}-E^{V}(m, N)-|\nabla f|^{2}\right) \chi \\
\geq & \left(\Sigma^{V}(m, N)-E^{V}(m, N)-\beta^{2}-o(1)\right) \chi^{2} \quad(R \rightarrow \infty) .
\end{aligned}
$$

In conjunction with (45) this shows that

$$
\left\|G \Phi_{m}\right\|^{2} \leq \frac{2 C}{\Sigma^{V}(m, N)-E^{V}(m, N)-\beta^{2}}\left\|\Phi_{m}\right\|^{2}
$$

for $R$ large enough. After letting $\varepsilon \rightarrow 0$ by monotone convergence a similar bound with $G$ replaced by $\chi \exp (\beta|X|)$ is obtained.

Proof of Theorem [6.1. This proof is a slight modification of the one in [8]. The basic idea is to show that there is effectively no interaction between localized particles and low momentum photons. To make this idea explicit we write our Hamiltonian in a gauge different from the usual Coulomb gauge.

To be precise, define

$$
\widetilde{A}(x)=A(x)-A(0),
$$

which is well defined owing to the ultraviolet cutoff. The unitary operator that accomplishes this is $U=\exp \left[i \sum_{j=1}^{N} \sqrt{\alpha} x_{j} \cdot A(0)\right]$. This is an 'operator-valued gauge transformation'. It commutes with $A(x)$ for all $x$, but not with $a_{\lambda}(k)$ or with $H_{f}$.

Define

$$
b_{\lambda}(k, X)=U a_{\lambda}(k) U^{*}=a_{\lambda}(k)-i w_{\lambda}(k, X),
$$

with $w_{\lambda}(k, X)=\chi_{\Lambda}(k)|k|^{-1 / 2} \varepsilon_{\lambda}(k) \cdot \sum_{j=1}^{N} x_{j}$. The transformed Hamiltonian $\widetilde{H}_{m}$ is

$$
\begin{gathered}
\widetilde{H}_{m}=U H_{m}^{V} U^{*}=\sum_{j=1}^{N}\left\{\left(p_{j}+\sqrt{\alpha} \widetilde{A}\left(x_{j}\right)\right)^{2}+\frac{g}{2} \sqrt{\alpha} \sigma_{j} \cdot B\left(x_{j}\right)\right\}+V+\widetilde{H}_{f}(m) \\
\widetilde{H}_{f}(m)=\sum_{\lambda=1,2} \int_{\mathbb{R}}^{3} \omega_{m}(k) b_{\lambda}^{*}(k, X) b_{\lambda}(k, X) .
\end{gathered}
$$

To estimate $\left\|a_{\lambda}(k) \Phi_{m}\right\|$, write

$$
a_{\lambda}(k) \Phi_{m}=U^{*} a_{\lambda}(k) \widetilde{\Phi}_{m}-i w_{\lambda}(k, X) \Phi_{m}
$$

where $\widetilde{\Phi}_{m}=U \Phi_{m}$, and note that

$$
\left\|w_{\lambda}(k, X) \Phi_{m}\right\| \leq \sqrt{N} \frac{\chi_{\Lambda}(k)}{|k|^{1 / 2}}\left\||X| \Phi_{m}\right\| .
$$


It remains to estimate $\left\|a_{\lambda}(k) \widetilde{\Phi}_{m}\right\|$. By the Schrödinger equation for $\widetilde{\Phi}_{m}$

$$
\begin{aligned}
\left(\widetilde{H}_{m}-E^{V}(m, N)\right) a_{\lambda}(k) \widetilde{\Phi}_{m}= & {\left[\widetilde{H}_{m}, a_{\lambda}(k)\right] \widetilde{\Phi}_{m} } \\
= & 2 \sqrt{\alpha}|k|^{-1 / 2} \varepsilon_{\lambda}(k) \cdot \sum_{j=1}^{N}\left(p_{j}+\sqrt{\alpha} \widetilde{A}\left(x_{j}\right)\right)\left(1-e^{-i k \cdot x_{j}}\right) \widetilde{\Phi}_{m} \\
& +i \frac{g}{2} \sqrt{\alpha} \frac{k \wedge \varepsilon_{\lambda}(k)}{\sqrt{|k|}} \cdot \sum_{j=1}^{N} \sigma_{j} e^{-i k \cdot x_{j}} \widetilde{\Phi}_{m} \\
& -\omega_{m}(k) b_{\lambda}(k, X) \widetilde{\Phi}_{m} .
\end{aligned}
$$

Now add $\omega_{m}(k) a_{\lambda}(k) \widetilde{\Phi}_{m}$ on both sides. Since $E^{V}(m, N)$ is the ground state energy and $\omega_{m}(k)>0$ the operator $\widetilde{H}_{m}-E^{V}(m, N)+\omega_{m}(k)$ has a bounded inverse $R\left(\omega_{m}(k)\right)$ and hence

$$
\begin{aligned}
a_{\lambda}(k) \widetilde{\Phi}_{m}= & 2 \sqrt{\alpha} R\left(\omega_{m}(k)\right) \frac{\chi_{\Lambda}(k)}{|k|^{1 / 2}} \varepsilon_{\lambda}(k) \cdot \sum_{j=1}^{N}\left(p_{j}+\sqrt{\alpha} \widetilde{A}\left(x_{j}\right)\right)\left(1-e^{-i k \cdot x_{j}}\right) \widetilde{\Phi}_{m} \\
& +i R\left(\omega_{m}(k)\right) \chi_{\Lambda}(k) \frac{g}{2} \sqrt{\alpha} \frac{k \wedge \varepsilon_{\lambda}(k)}{\sqrt{|k|}} \cdot \sum_{j=1}^{N} \sigma_{j} e^{-i k \cdot x_{j}} \widetilde{\Phi}_{m} \\
& -R\left(\omega_{m}(k)\right) \omega_{m}(k) i w_{\lambda}(k, X) \widetilde{\Phi}_{m}
\end{aligned}
$$

For consistency, note that for $|k|>\Lambda w_{\lambda}(k, X)=0$ and hence $a_{\lambda}(k) \widetilde{\Phi}_{m}=0$, i.e, for these modes $\widetilde{\Phi}_{m}$ is the vacuum as it should be for a minimizer. Since $\left\|R\left(\omega_{m}(k)\right)\right\| \leq \omega_{m}(k)^{-1}$ the norm of the last term is bounded by

$$
\sqrt{\alpha} \sqrt{N} \frac{\chi_{\Lambda}(k)}{|k|^{1 / 2}}\left\||X| \widetilde{\Phi}_{m}\right\| .
$$

To bound the norm of the first term we need to estimate

$$
\begin{aligned}
& \left\|\sum_{j=1}^{N} R\left(\omega_{m}(k)\right) \varepsilon_{\lambda}(k) \cdot\left(p_{j}+\sqrt{\alpha} \widetilde{A}\left(x_{j}\right)\right)\left(1-e^{-i k \cdot x_{j}}\right) \widetilde{\Phi}_{m}\right\| \\
= & \sup _{\|\eta\| \leq 1}\left|\sum_{j=1}^{N}\left(\varepsilon_{\lambda}(k) \cdot\left(p_{j}+\sqrt{\alpha} \widetilde{A}\left(x_{j}\right)\right) R\left(\omega_{m}(k)\right) \eta,\left(1-e^{-i k \cdot x_{j}}\right) \widetilde{\Phi}_{m}\right)\right| \\
\leq & \sup _{\|\eta\| \leq 1}\left[\sum_{j=1}^{N}\left\|\left(p_{j}+\sqrt{\alpha} \widetilde{A}\left(x_{j}\right)\right) R\left(\omega_{m}(k)\right) \eta\right\|^{2}\right]^{1 / 2}\left[\sum_{j=1}^{N}\left\|\left(1-e^{-i k \cdot x_{j}}\right) \widetilde{\Phi}_{m}\right\|^{2}\right]^{1 / 2}
\end{aligned}
$$

Next estimate the square of the first factor to get

$$
\begin{aligned}
& \left(\eta, R\left(\omega_{m}(k)\right)\left[\sum_{j=1}^{N}\left(p_{j}+\sqrt{\alpha} \widetilde{A}\left(x_{j}\right)\right)^{2}\right] R\left(\omega_{m}(k)\right) \eta\right) \\
\leq & a\left(\eta, R\left(\omega_{m}(k)\right) H_{m}^{V} R\left(\omega_{m}(k)\right) \eta\right)+b \\
\leq & a\left(\eta, R\left(\omega_{m}(k)\right) \eta\right)+\left(a E^{V}(m, N)+b\right)\left(\eta, R\left(\omega_{m}(k)\right)^{2} \eta\right) \\
\leq & C \frac{(\Lambda+1)}{|k|^{2}} \text { for }|k| \leq \Lambda
\end{aligned}
$$


where $a$ and $b$ are independent of $m$. Since $\sup _{m<1} E^{V}(m, N)<\infty$ the constant $C$ is also independent of $m$. Finally the second factor in (55) is bounded by $|k|\left\||X| \widetilde{\Phi}_{m}\right\|$. The term containing the Pauli matrices in (53) is estimated similarly. In conjuction with (53), (52)(55) and (56) this shows that

$$
\left\|a_{\lambda}(k) \Phi_{m}\right\| \leq C \sqrt{\alpha}(\Lambda+1)^{1 / 2} \frac{\chi_{\Lambda}(k)}{|k|^{1 / 2}}\left\||X| \widetilde{\Phi}_{m}\right\|
$$

This, together with Lemma 6.2, proves the theorem.

Next we differentiate (53) with respect to $k$. There is a slight problem with this calculation since the polarization vectors cannot be defined in a smooth fashion globally. We make the following choice for the polarization vectors.

$$
\varepsilon_{1}(k)=\frac{\left(k_{2},-k_{1}, 0\right)}{\sqrt{k_{1}^{2}+k_{2}^{2}}}
$$

and

$$
\varepsilon_{2}(k)=\frac{k}{|k|} \wedge \varepsilon_{1}(k)
$$

6.3. THEOREM (Photon derivative bound). Assume that there is binding, i.e., $\Sigma^{V}(m, N)-$ $E^{V}(m, N)>0$. Assume that $\Phi_{m}$ is a normalized ground state for the many-body Hamiltonian $H_{m}^{V}$, $m \geq 0$. Then for $|k|<\Lambda$ and $\left(k_{1}, k_{2}\right) \neq(0,0)$

$$
\left\|\nabla_{k} a_{\lambda}(k) \Phi_{m}\right\|<\frac{Q \sqrt{\alpha}(1+|g|)}{|k|^{1 / 2} \sqrt{k_{1}^{2}+k_{2}^{2}}},
$$

where $Q$ is a finite constant independent of $\Phi_{m}, g, \alpha, \Lambda$, and depends on $m$ only through the binding energy $\Sigma^{V}(m, N)-E^{V}(m, N)>0$.

Proof. We differentiate 53 with respect to $k$ and obtain

$$
\begin{aligned}
& \nabla_{k}\left(a_{\lambda}(k)\right) \widetilde{\Phi}_{m}= \\
& 2 \sqrt{\alpha} R\left(\omega_{m}(k)\right)^{2} \frac{k}{|k|} \varepsilon_{\lambda}(k) \cdot \sum_{j=1}^{N}\left(p_{j}+\sqrt{\alpha} \widetilde{A}\left(x_{j}\right)\right) \frac{\left(1-e^{-i k \cdot x_{j}}\right)}{|k|^{1 / 2}} \widetilde{\Phi}_{m}+ \\
& 2 \sqrt{\alpha} R\left(\omega_{m}(k)\right) \nabla_{k}\left(\varepsilon_{\lambda}(k)\right) \cdot \sum_{j=1}^{N}\left(p_{j}+\sqrt{\alpha} \widetilde{A}\left(x_{j}\right)\right) \frac{\left(1-e^{-i k \cdot x_{j}}\right)}{|k|^{1 / 2}} \widetilde{\Phi}_{m}+ \\
& 2 \sqrt{\alpha} R\left(\omega_{m}(k)\right) \varepsilon_{\lambda}(k) \cdot \sum_{j=1}^{N}\left(p_{j}+\sqrt{\alpha} \widetilde{A}\left(x_{j}\right)\right) \nabla_{k}\left(\frac{\left(1-e^{-i k \cdot x_{j}}\right)}{|k|^{1 / 2}}\right) \widetilde{\Phi}_{m} \\
& +\frac{g}{2} \sqrt{\alpha} \nabla_{k}\left(i R\left(\omega_{m}(k)\right) \frac{k \wedge \varepsilon_{\lambda}(k)}{\sqrt{|k|}} \cdot \sum_{j=1}^{N} \sigma_{j} e^{-i k \cdot x_{j}} \widetilde{\Phi}_{m}\right) \\
& -\nabla_{k}\left(R\left(\omega_{m}(k)\right) \omega_{m}(k) i w_{\lambda}(k, X) \widetilde{\Phi}_{m}\right) \cdot
\end{aligned}
$$


The norms of the first and third terms can estimated precisely the same way as in (55) and (56), and yields a bound of the form

$$
\frac{C \sqrt{\alpha}}{|k|^{3 / 2}}\left\|(1+|X|) \widetilde{\Phi}_{m}\right\|
$$

For the second term, a straightforward calculation shows that

$$
\left|\nabla_{k} \varepsilon_{i}(k)\right| \leq \frac{\text { const. }}{\sqrt{k_{1}^{2}+k_{2}^{2}}} \text { for } i=1,2 .
$$

The last term is dealt with in a similar fashion as the previous ones. Using the steps in (55) and (56), this leads to the bound

$$
\left\|\nabla_{k}\left(a_{\lambda}(k)\right) \widetilde{\Phi}_{m}\right\| \leq \frac{C \sqrt{\alpha}}{|k|^{1 / 2} \sqrt{k_{1}^{2}+k_{2}^{2}}}\left\|(1+|X|) \widetilde{\Phi}_{m}\right\| .
$$

The fourth term can be estimated in the same fashion to yield a similar result.

Differentiating (51) leads to the same estimate with $\widetilde{\Phi}_{m}$ replaced by $\Phi_{m}$. This, together with Lemma 6.2, proves the theorem.

\section{PROOF OF THEOREM 2.1}

The proof will be done in two steps.

Proof. Step 1. The Hamiltonian $H_{m}^{V}$ has a normalized ground state $\Phi_{m}$, by Theorem 4.1. Pick a sequence $m_{1}>m_{2}>\ldots$ tending to zero and denote the corresponding eigenvectors by $\Phi_{j}$. This sequence is a minimizing sequence for $H_{0}^{V}$ by Theorem 5.3. Since $\left\|\Phi_{j}\right\|$ is bounded there is a subsequence (call it again $\Phi_{j}$ ) which has a weak limit $\Phi$. Since $H_{0}^{V}-E^{V}(0, N) \geq 0$ and by the lower semi-continuity of non-negative quadratic forms (in our case, $H_{0}^{V}-E^{V}(0, N)$ )

$$
0 \leq\left(\Phi,\left(H_{0}^{V}-E^{V}(0, N)\right) \Phi\right) \leq \liminf _{j \rightarrow \infty}\left(\Phi_{j},\left(H_{0}^{V}-E^{V}(0, N)\right) \Phi_{j}\right)=0 .
$$

Hence $\Phi$ will be a (normalized) ground state if we show that $\|\Phi\|=1$ (i.e. $\Phi_{j} \rightarrow \Phi$ strongly). It is important to note, however that if we write $\Phi_{j}=\left\{\Phi_{j, 0}, \Phi_{j, 1}, \ldots, \Phi_{j, n}, \ldots\right\}$, where $\Phi_{j, n}$ is the $n$-photon component of $\Phi_{j}$ then it suffices to prove the $L^{2}$ norm-convergence of each $\Phi_{j, n}$. The reason is the uniform bound on the total average photon number; see the remark after Theorem 6.1 which implies

$$
\sum_{n \geq N}\left\|\Phi_{j, n}\right\|^{2} \leq \text { const } N^{-1}
$$

Likewise, it suffices to prove the strong $L^{2}$ convergence in the bounded domain in which $|X|<R$ for each finite $R$. The reason for this is the exponential decay given in Lemma 6.2, which is uniform by Lemma 5.4. Finally, by Theorem 6.1 $\Phi_{j, n}\left(X, k_{1}, \ldots, k_{n}\right)$ vanishes if $\left|k_{i}\right|>\Lambda$ for some $i$. So it suffices to show $L^{2}$ convergence for $\Phi_{j, n}$ restricted to

$$
\Omega=\left\{\left(X, k_{1}, \ldots, k_{n}\right):|X|<R ;\left|k_{i}\right|<\Lambda, i=1, \ldots, n\right\} \subset \mathbb{R}^{3(N+n)}
$$


for each $R>0$.

Step 2. For each $p<2$ and $R>0$ we show that $\Phi_{j, n}$ restricted to $\Omega$ is a bounded sequence in $W^{1, p}(\Omega)$. The key to this bound is $(60)$ and

$$
\left(a_{\lambda}(k) \Phi_{j}\right)_{n-1}\left(X, k_{1}, \ldots, k_{n-1}\right)=\sqrt{n} \Phi_{j, n}\left(X, k, k_{1}, \ldots, k_{n-1}\right)
$$

where the arguments $\lambda, \lambda_{1}, \ldots, \lambda_{n-1}$ and the spin indices have been supressed. By the symmetry of $\Phi_{j, n}$, (64), Hölder's inequality and (60)

$$
\begin{aligned}
& \int_{B_{R}} d X \int_{\left|k_{1}\right|, \ldots,\left|k_{n}\right|<\Lambda} d k_{1} \ldots d k_{n} \sum_{i=1}^{n}\left|\nabla_{k_{i}} \Phi_{j, n}\left(X, k_{1}, \ldots, k_{n}\right)\right|^{p} \\
& =n^{1-p / 2} \int_{B_{R}} d X \int_{\left|k_{1}\right|, \ldots,\left|k_{n}\right|<\Lambda} d k_{1} \ldots d k_{n}\left|\nabla_{k_{1}}\left(a_{\lambda}\left(k_{1}\right) \Phi_{j}\right)_{n-1}\left(X, k_{2}, \ldots, k_{n}\right)\right|^{p} \\
& \leq C \int_{\left|k_{1}\right|<\Lambda} d k_{1}\left(\int_{B_{R}} d X \int_{\left|k_{2}\right|, \ldots,\left|k_{n}\right|<\Lambda} d k_{2} \ldots d k_{n}\left|\nabla_{k_{1}}\left(a_{\lambda}\left(k_{1}\right) \Phi_{j}\right)_{n-1}\left(X, k_{2}, \ldots, k_{n}\right)\right|^{2}\right)^{p / 2} \\
& \leq C \int_{\left|k_{1}\right|<\Lambda} d k_{1}\left\|\nabla_{k_{1}} a_{\lambda}\left(k_{1}\right) \Phi_{j}\right\|^{p} \leq \mathrm{const}
\end{aligned}
$$

independent of $j$. The constant $C$ depends on all the parameters, but is finite because $\left|k_{i}\right| \leq \Lambda$ in the integration. Similarly, by Hölder's inequality

$$
\begin{aligned}
\left\|\chi(|X|<R) \nabla_{X} \Phi_{j, n}\right\|_{p}^{p} & \leq C\left\|\chi(|X|<R) \nabla_{X} \Phi_{j, n}\right\|_{2}^{p} \\
& \leq C\left(\Phi_{j}, \sum_{i=1}^{N} p_{i}^{2} \Phi_{j}\right)^{p / 2}
\end{aligned}
$$

which is uniformly bounded by Lemma (A.5).

Since the classical derivative of $a_{\lambda}(k) \Phi_{m}$ is not defined in all of $\Omega$ one has to check that the weak derivative coincides with the classical derivative a.e.. Because of our definitions (58) and (59), the classical derivative is not defined along the 3 -axis.

One has to show that

$$
\int_{\Omega} \partial_{i} \psi \Phi_{j, n}=\lim _{\varepsilon \rightarrow 0} \int_{\Omega_{\varepsilon}} \partial_{i} \psi \Phi_{j, n}=-\lim _{\varepsilon \rightarrow 0} \int_{\Omega_{\varepsilon}} \psi \partial_{i} \Phi_{j, n} \quad i=1, \ldots, 3(N+n) .
$$

for any test function $\psi \in C_{c}^{\infty}(\Omega)$. Here $\Omega_{\varepsilon}$ is $\Omega$ with an $\varepsilon$ cylinder around the 3 -axis removed in each $k$-ball. The first equality is trivial; it is the second equality that has to be checked. This amounts to showing that the boundary term, coming from the integration by parts vanishes in the limit as $\varepsilon$ tends to zero. But this follows immediately from Theorem 6.1.

This shows that $\Phi_{j, n}$ as a function of all its $3(N+n)$ variables, is in the Sobolev space $W^{1, p}(\Omega)$ and that $\sup _{j}\left\|\Phi_{j, n}\right\|_{W^{1, p}(\Omega)}<\infty$. Since $\Phi_{n, j}$ converges weakly in $L^{2}(\Omega)$ it converges weakly in $L^{p}(\Omega)$ and since the sequence is bounded in $W^{1, p}(\Omega), \nabla \Phi_{n, j}$ converges weakly to $\nabla \Phi_{n}$.

The Rellich-Kondrachov theorem (see 18 Theorem 8.9) states that such a sequence converges strongly in $L^{q}(\Omega)$ if $1 \leq q<[3 p(N+n) / 3(N+n)-p]$. The boundedness of $\Omega$ is crucial here. For 
our purposes we need $q=2$, and hence we have to pick $p$ such that

$$
2>p>\frac{2 \cdot 3(N+n)}{2+3(N+n)}
$$

which is possible for each $N$ and $n$. We conclude that $\Phi_{j, n} \rightarrow \Phi_{n}$ strongly in $L^{2}(\Omega)$ as $j \rightarrow \infty$, for each $n$ and $R$. This proves the theorem.

Remark: Theorem 6.3 essentially says that the derivative is almost, but not quite in $L^{2}$. For high dimensions, $3(N+n)$, the required $p$ is as close as we please to 2 if we require $q=2$, but $p=2$ is not allowed. The way out of the difficulty was to prove a uniform bound on the number operator and use this to say that that it suffices to prove strong convergence for each $n$ separately. With $n$ then fixed, it is possible to find a $p<2$ that yields $q=2$. It is, therefore, crucial to have the derivative in every $L^{p}$ space with $p<2$. The resolution of the problem of the infrared singularity is thus seen to be a delicate matter.

\section{A Appendix: LOCALIZATION ESTIMATES}

In this appendix we collect a few facts which we use several times in this paper. Generally we worry about localizations of Hamiltonians in configuration space. While this is standard for Schrödinger operators it is somewhat more complicated in the presence of the radiation field. This is chiefly due to the problem of localization of photons.

We begin by stating a few well known facts about partitions of unity. Let $\beta$ denote one of the $2^{N}$ subsets of the set of integers $1,2, \ldots, N$. Its complement is denoted by $\beta^{c}$. As shown in 16 there exists a family of smooth functions $j_{\beta}$ having the following four properties.

(i)

$$
\sum_{\beta} j_{\beta}^{2}=1
$$

(ii) For $\beta \neq\{1, \ldots, N\}$ (including the empty set) the $j_{\beta}$ 's are homogeneous of degree 0 and live outside the ball of radius $R$ centered at the origin and

$$
\operatorname{supp} j_{\beta} \subset\left\{X: \min _{i \in \beta, j \in \beta^{c}}\left(\left|x_{i}-x_{j}\right|,\left|x_{j}\right|\right) \geq c|X|\right\},
$$

where $C$ is some positive constant.

(iii) In the case where $\beta=\{1, \ldots, N\}, j_{\beta}$ is compactly supported.

Corresponding to these electron localizations we define photon localizations.

For given $\beta \neq\{1, \ldots, N\}$ consider the function

$$
g_{1}(y ; \beta, X)=\Pi_{j \in \beta^{c}}\left(1-\chi\left(\frac{y-x_{j}}{P}\right)\right)
$$

where $\chi$ is a smoothed characteristic function of the unit ball. Define $g_{2}(y ; \beta, X)=1-g_{1}(y, \beta, X)$. In the variable $y$, the function $g_{1}$ is supported away from the particles in $\beta^{c}$ while $g_{2}$ lives close to 
the particles in $\beta^{c}$. Next define, for $i=1,2$,

$$
j_{i}(y ; \beta, X)=\frac{g_{i}(y ; \beta, X)}{\sqrt{g_{1}(y ; \beta, X)^{2}+g_{2}(y ; \beta, X)^{2}}} .
$$

Certainly $j_{1}^{2}+j_{2}^{2}=1$ and a simple computation shows that

$$
\left|\nabla j_{i}\right| \leq \frac{\text { const. }}{P}
$$

In the case where $\beta=\{1, \ldots, N\}$ the construction of $j_{1}$ and $j_{2}$ is similar to the above one except that the function $g_{1}$ depends on $y$, is equal to one in a neighborhood of the origin and is compactly supported.

With the help of $j_{1}$ and $j_{2}$ the photons can now be localized as was done in Section 4 . Let $U_{\beta}(X) \mathcal{F} \rightarrow \mathcal{F} \otimes \mathcal{F}$ be the corresponding isometric transformation, i.e., the one that is defined via the relation

$$
U_{\beta}(X) a^{\#}(h) U_{\beta}^{*}(X)=a^{\#}\left(j_{1} h\right) \otimes \mathcal{I}+\mathcal{I} \otimes a^{\#}\left(j_{2} h\right)
$$

The tensor product indicated is a tensor product between Fock spaces.

We denote by $H_{\beta}$ the Hamiltonian of the form (4) with photon mass, but only for the particles in the set $\beta$. More precisely this operator acts on $L^{2}\left(\mathbb{R}^{3|\beta|}\right) \otimes \mathcal{F}$. By $H^{\beta^{c}}$ we denote the Hamiltonian of the form (4) with photon mass, but only for the particles in the set $\beta^{c}$ where the interaction with the nuclei has been dropped. This operator acts on $L^{2}\left(\mathbb{R}^{3\left|\beta^{c}\right|}\right) \otimes \mathcal{F}$ In particular we keep the interaction among those particles. In the case where $\beta=\{1, \ldots, N\}$ the Hamiltonian $H^{\beta^{c}}=H_{f}(m)$.

\section{A.1. LEMMA (Localization of Hamiltonian). For every $\beta$}

$$
j_{\beta} H j_{\beta}=U_{\beta}^{*}(X) j_{\beta}\left[H_{\beta} \otimes \mathcal{I}+\mathcal{I} \otimes H^{\beta^{c}}\right] j_{\beta} U_{\beta}(X)+o(1) .
$$

For $\beta \neq\{1, \ldots, N\}, o(1) \rightarrow 0$ as first $R \rightarrow \infty$ and then $P \rightarrow \infty$. If $\beta=\{1, \ldots, N\}$ then $o(1) \rightarrow 0$ as $P \rightarrow \infty$ for every fixed $R>0$.

Proof. Our immediate aim is to compare the field energy $H_{f}$ with the localized field energy $U_{\beta}^{*}(X)\left[H_{f} \otimes \mathcal{I}+\mathcal{I} \otimes H_{f}\right] U_{\beta}(X)$. For simplicity the various indices are supressed and $U_{\beta}(X)$ is replaced by $U_{\beta}$. The variable $X$ plays no role here. Pick an orthonormal basis $\left\{g_{j}\right\}_{j=1}^{\infty}$ of $L^{2}\left(\mathbb{R}^{3}\right)$ in $H^{1 / 2}\left(\mathbb{R}^{3}\right)$. States of the form

$$
\zeta=\text { const. } a_{\lambda_{i_{1}}}^{*}\left(g_{i_{1}}\right) \cdots a_{\lambda_{i_{k}}}^{*}\left(g_{i_{k}}\right) \mid 0>
$$

where $k$ is finite, form an orthonormal basis in the Fock space. The field energy acts on such states as

$$
H_{f} \zeta=\sum_{j=1}^{k} a_{\lambda_{i_{1}}}^{*}\left(h_{i_{1}}\right) \cdots a_{\lambda_{i_{j}}}^{*}\left(\omega h_{i_{j}}\right) \cdots a_{\lambda_{i_{k}}}^{*}\left(h_{i_{k}}\right) \mid 0>
$$


Thus, we have that

$$
H_{f} \zeta=U_{\beta}^{*} \sum_{j=1}^{k} c_{\lambda_{i_{1}}}^{*}\left(h_{i_{1}}\right) \cdots c_{\lambda_{i_{j}}}^{*}\left(\omega h_{i_{j}}\right) \cdots c_{\lambda_{i_{k}}}^{*}\left(h_{i_{k}}\right) U_{\beta} \mid 0>
$$

and

$$
H_{f}=U_{\beta}^{*}\left[H_{f} \otimes \mathcal{I}+\mathcal{I} \otimes H_{f}\right] U_{\beta}+E_{f}
$$

where the error $E_{f}$ is given by

$$
\begin{gathered}
E_{f} \zeta=U_{\beta}^{*} \sum_{j=1}^{k} c_{\lambda_{i_{1}}}^{*}\left(h_{i_{1}}\right) \cdots\left(a_{\lambda_{i_{j}}}^{*}\left(\left[j_{1}, \omega\right] h_{i_{j}}\right) \otimes \mathcal{I}+\mathcal{I} \otimes a_{\lambda_{i_{j}}}^{*}\left(\left[j_{2}, \omega\right] h_{i_{j}}\right)\right) \\
\cdots c_{\lambda_{i_{k}}}^{*}\left(h_{i_{k}}\right) U_{\beta} \mid 0>
\end{gathered}
$$

Thus $E_{f}$ is given by the operator (the $\lambda$ 's are omitted)

$$
E_{f}=U_{\beta}^{*} \sum_{k}\left[a^{*}\left(\left[\omega, j_{1}\right] g_{k}\right) \otimes \mathcal{I}+\mathcal{I} \otimes a^{*}\left(\left[\omega, j_{2}\right] g_{k}\right)\right]\left[a\left(j_{1} g_{k}\right) \otimes \mathcal{I}+\mathcal{I} \otimes a\left(j_{2} g_{k}\right)\right] U_{\beta}
$$

Standard estimates lead to

$$
\left\|E_{f} \Psi\right\| \leq\left(\left\|\left[\omega, j_{1}\right]\right\|+\left\|\left[\omega, j_{2}\right]\right\|\right)\|\sqrt{\mathcal{N}+1} \Psi\|
$$

where $\mathcal{N}$ is the number operator.

Here $\left\|\left[j_{1}, \omega\right]\right\|$ denotes the operator norm associated with the kernel $\left[j_{1}, \omega\right]$. This norm can be estimated using the formula

$$
\left[j_{1}, \omega\right]=\left[j_{1}, \omega^{2}\right] \frac{1}{\omega}+\omega^{2}\left[j_{1}, \frac{1}{\omega}\right] .
$$

Recalling the definition of $j_{1}$, the operator norm of the first term is easily seen to be bounded by a const./P. Likewise, the second term, using the formula

$$
\frac{1}{\sqrt{p^{2}+m^{2}}}=\frac{1}{\pi} \int_{0}^{\infty} \frac{1}{t+p^{2}+m^{2}} \frac{d t}{\sqrt{t}},
$$

can be estimated by const./P. The term $\left\|\left[j_{2}, \omega\right]\right\|$ is estimated in a similar fashion. The estimate (80) immediately shows that for a general state $\Phi$ we have that

$$
\left|\left(\Phi,\left[H_{f}-U_{\beta}^{*}\left[H_{f} \otimes \mathcal{I}+\mathcal{I} \otimes H_{f}\right] U_{\beta}\right] \Phi\right)\right| \leq \frac{\text { const. }}{P}(\Phi, \mathcal{N} \Phi) .
$$

Since the photons have a mass we can estimate the number operator in terms of the field energy. The field energy is relatively bounded with respect to the Hamiltonian, i.e., $H_{f} \leq a H_{m}^{V}+b$ for some positive constants $a$ and $b$, and thus we obtain

$$
\left|\left(\Phi,\left[H_{f}-U_{\beta}^{*}\left[H_{f} \otimes \mathcal{I}+\mathcal{I} \otimes H_{f}\right] U_{\beta}\right] \Phi\right)\right| \leq \frac{\text { const. }}{P m}\left(\Phi,\left[a H_{m}^{V}+b\right] \Phi\right) .
$$

Note that this estimate had nothing to do with the electron, in particular the $x$-space cutoff is not present in the calculation. 
Next we have to compare $\sum_{j=1}^{N}\left(p_{j}+\sqrt{\alpha} A\left(x_{j}\right)\right)^{2}$ with

$$
U_{\beta}^{*}(X) j_{\beta}\left[\sum_{i \in \beta}\left(p_{i}+\sqrt{\alpha} A\left(x_{i}\right)\right)^{2} \otimes \mathcal{I}+\mathcal{I} \otimes \sum_{j \in \beta^{c}}\left(p_{j}+\sqrt{\alpha} A\left(x_{j}\right)\right)^{2}\right] j_{\beta} U_{\beta}(X) .
$$

This time the $X$-space cutoff is important. We would like to estimate the difference

$$
\begin{gathered}
j_{\beta} \sum_{i \in \beta}\left[\left(p_{i}+\sqrt{\alpha} A\left(x_{i}\right)\right)^{2}-U_{\beta}^{*}(X)\left(p_{i}+\sqrt{\alpha} A\left(x_{i}\right)\right)^{2} \otimes \mathcal{I} U_{\beta}(X)\right] j_{\beta} \\
+\quad j_{\beta} \sum_{i \in \beta^{c}}\left[\left(p_{i}+\sqrt{\alpha} A\left(x_{i}\right)\right)^{2}-U_{\beta}^{*}(X) \mathcal{I} \otimes\left(p_{i}+\sqrt{\alpha} A\left(x_{i}\right)\right)^{2} U_{\beta}(X)\right] j_{\beta} .
\end{gathered}
$$

It suffices to treat the first term, the other is similar. It can be easily expressed as

$$
j_{\beta}\left[\sum_{i \in \beta}\left(p_{i}+\sqrt{\alpha} A\left(x_{i}\right)\right) Q_{i}+Q_{i}\left(p_{i}+\sqrt{\alpha} A\left(x_{i}\right)\right)-Q_{i}^{2}\right] j_{\beta}
$$

where

$$
Q_{i}=p_{i}+\sqrt{\alpha} A\left(x_{i}\right)-U_{\beta}^{*}(X)\left(p_{i}+\sqrt{\alpha} A\left(x_{i}\right)\right) \otimes \mathcal{I} U_{\beta}(X) .
$$

Using the form boundedness of the kinetic energy with respect to the full Hamiltonian, we have

$$
\left(\Psi, \sum_{j=1}^{N}\left(p_{j}+\sqrt{\alpha} A\left(x_{j}\right)\right)^{2} \Psi\right) \leq a\left(\Psi, H_{m}^{V} \Psi\right)+b(\Psi, \Psi)
$$

for positive constants $a$ and $b$.. Thus, using Schwarz' inequality it suffices to show that

$$
\left\|Q_{i} j_{\beta} \Psi\right\|=o(1) \text { for } i \in \beta,
$$

as $R$ (the localization radius for the electrons) tends to infinity. Denote by

$$
h_{i, x}^{\lambda}(y)=(2 \pi)^{-3 / 2} \int_{|k|<\Lambda} \frac{1}{\omega(k)} \varepsilon_{i}^{\lambda}(k) e^{i k \cdot(y-x)} d^{3} k .
$$

Explicitly, $Q_{i}$ is given by

$$
\begin{aligned}
& p_{i}-U_{\beta}^{*}(X) p_{i} \otimes \mathcal{I} U_{\beta}(X) \\
+ & U_{\beta}^{*}(X)\left[\sum_{\lambda} a_{\lambda}\left(\left[j_{1}-1\right] h_{x}^{\lambda}\right) \otimes \mathcal{I}+\mathcal{I} \otimes a_{\lambda}\left(j_{2} h_{x}^{\lambda}\right)\right] U_{\beta}(X) \\
+ & U_{\beta}^{*}(X)\left[\sum_{\lambda} a_{\lambda}^{*}\left(\left[j_{1}-1\right] h_{x}^{\lambda}\right) \otimes \mathcal{I}+\mathcal{I} \otimes a_{\lambda}^{*}\left(j_{2} h_{x}^{\lambda}\right)\right] U_{\beta}(X),
\end{aligned}
$$

and it suffices to estimate each of these terms separately. Each of the last two terms can be brought into the form

$$
\left\|U_{\beta}^{*}(X) a^{\#}(f) \otimes \mathcal{I} U_{\beta}(X) j_{\beta} \Psi\right\|
$$


where $f$ is one of the functions

$$
\left[j_{1}(y, \beta, X)-1\right] h_{1, x_{j}}^{\lambda}(y) \text { or } j_{2}(y, \beta, X) h_{1, x_{j}}^{\lambda}(y) \quad j \in \beta \text {. }
$$

The terms (92) are estimated by

$$
\sup _{X}\left\{j_{\beta}(X)\left\|\left[j_{1}-1\right] h_{i, x_{j}}^{\lambda}\right\|_{2}\right\} \sqrt{(\Psi,(\mathcal{N}+1) \Psi)}
$$

respectively

$$
\sup _{X}\left\{j_{\beta}(X)\left\|j_{2} h_{1, x_{j}}^{\lambda}\right\|_{2}\right\} \sqrt{(\Psi,(\mathcal{N}+1) \Psi)} .
$$

In both formulas the index $j$ is in $\beta$. The function $j_{\beta}$ lives in the region where $\left|x_{i}-x_{j}\right| \geq c R$ for $i \in \beta$ and $j \in \beta^{c}$. The function $j_{1}-1$ (and likewise $j_{2}$ ) is not zero only if $\left|y-x_{j}\right| \leq P$ for some $j \in \beta^{c}$. Thus, $j_{\beta}(X)\left(j_{1}-1\right)(y)$ and $j_{\beta}(X) j_{2}(y)$ are nonzero only if $\left|y-x_{i}\right| \geq c R-P$. As $c R-P$ gets large only the tail of the function $h^{\lambda}$ contributes to the integral which can be made as small as we please. The number operator is bounded by the field energy times $1 / m$ which in turn is bounded by the full energy.

To estimate the first term in (91) we calculate

$$
\begin{aligned}
& p_{i}-U_{\beta}^{*}(X) p_{i} \otimes \mathcal{I} U_{\beta}(X)= \\
& U_{\beta}^{*}(X) \sum_{k}\left[a^{*}\left(\left[p_{i}, j_{1}\right] g_{k}\right) \otimes \mathcal{I}+\mathcal{I} \otimes a^{*}\left(\left[p_{i}, j_{2}\right] g_{k}\right)\right] \times \\
& {\left[a\left(j_{1} g_{k}\right) \otimes \mathcal{I}+\mathcal{I} \otimes a\left(j_{2} g_{k}\right)\right] U_{\beta}(X) .}
\end{aligned}
$$

Note that the tensor product in the first line is different from the second. In the first the identity acts on $L^{2}\left(\mathbb{R}^{3\left|\beta^{c}\right|}\right) \otimes \mathcal{F} \otimes \mathcal{F}$ while in the second $\otimes$ indicates the tensor product of the Fock spaces only. The functions $g_{k}$ indicates a basis of $L^{2}\left(\mathbb{R}^{3}\right)$. The operators $U_{\beta}^{*}(X)$ and $U_{\beta}(X)$ have unit norm. Thus

$$
\begin{aligned}
& \| U_{\beta}^{*}(X) \sum_{k}\left[a^{*}\left(\left[p_{i}, j_{1}\right] g_{k}\right) \otimes \mathcal{I}+\mathcal{I} \otimes a^{*}\left(\left[p_{i}, j_{2}\right] g_{k}\right)\right] \times \\
& {\left[a\left(j_{1} g_{k}\right) \otimes \mathcal{I}+\mathcal{I} \otimes a\left(j_{2} g_{k}\right)\right] U_{\beta}(X) \Psi \|} \\
& \leq\left(\left\|\left[p_{i}, j_{1}\right]\right\|+\left\|\left[p_{i}, j_{2}\right]\right\|\right)\|\sqrt{\mathcal{N}+1} \Psi\|,
\end{aligned}
$$

where $\|\cdot\|$ indicates that the operator norm has been taken. The norms of the commutators are of the order $1 / P$ and hence vanish as $P \rightarrow \infty$. Since the photons have a mass we can estimate the number operator in terms of the field energy.

Similar consideration apply to the $\beta^{c}$ term in (85). The only difference is that instead of (94) and (95) we have

$$
\sup _{X}\left\{j_{\beta}(X)\left\|j_{1} h_{x_{j}}^{\lambda}\right\|_{2}\right\} \sqrt{(\Psi,(\mathcal{N}+1) \Psi)}
$$

respectively

$$
\sup _{X}\left\{j_{\beta}(X)\left\|\left[j_{2}-1\right] h_{x_{j}}^{\lambda}\right\|_{2}\right\} \sqrt{(\Psi,(\mathcal{N}+1) \Psi)},
$$


with $j \in \beta^{c}$. Again this terms tend to zero as $P \rightarrow \infty$. The proof for the case where $\beta=\{1, \ldots, N\}$ is similar but simpler since the operator $U_{\beta}$ does not depend on $X$.

Finally, we have to compare the $\sigma \cdot B$ term with its localized counterparts. The estimates are similar to, but much easier than the estimates for $(p+\sqrt{\alpha} A(x))^{2}$ and are omitted for the convenience of the reader and authors who, by now, are exhausted.

A simple consequence of Lemma A.1 is the following.

A.2. COROLLARY. Let $\phi$ be a smooth function on $\mathbb{R}^{3 N}$ such that $j_{\beta} \phi \equiv 0$ for $\beta=\{1, \ldots, N\}$. Thus, $\phi$ depends on $R$. Then, as operators,

$$
\phi H \phi \geq\left(\Sigma^{V}(m, N)+o(1)\right) \phi^{2}
$$

Here, $\Sigma^{V}(m, N)=\min _{1 \leq N^{\prime}<N}\left(E^{V}\left(N^{\prime}\right)+E^{0}\left(N-N^{\prime}\right)\right)$ and $o(1)$ vanishes as $R \rightarrow \infty$.

Proof. By the IMS localization formula we have that

$$
\phi H \phi=\sum_{\beta} \phi j_{\beta} H j_{\beta} \phi-\phi^{2} \sum_{\beta}\left|\nabla j_{\beta}\right|^{2}
$$

where the second term goes to 0 as $R \rightarrow \infty$. With our assumption on $\phi$ only the sets $\beta$ with $\beta^{c} \neq \emptyset$ contribute. From Lemma A.1 we get that

$$
\phi H \phi=\sum_{\beta} U_{\beta}^{*}(X) \phi j_{\beta}\left[H_{\beta} \otimes \mathcal{I}+\mathcal{I} \otimes H^{\beta^{c}}\right] j_{\beta} \phi U_{\beta}(X)+o(1)
$$

as first $R \rightarrow \infty$ then $P \rightarrow \infty$. Certainly $H_{\beta} \geq E^{V}(m,|\beta|)$ and $H^{\beta^{c}} \geq E^{0}\left(m,\left|\beta^{c}\right|\right)$ from which the statement immediately follows.

A.3. LEMMA. Let $\Psi_{n}$ be a normalized sequence in $\mathcal{H}$ whose energy is uniformly bounded and such that for any $\Phi \in \mathcal{H}$ with finite energy,

$$
\left(\Psi_{n}, \Phi\right) \rightarrow 0 \text {, and }\left(\Psi_{n}, H \Phi\right) \rightarrow 0
$$

Then

$$
\left(\phi_{R} \Psi_{n}, U^{*} \mathcal{I} \otimes P_{2} U \phi_{R} \Psi_{n}\right) \rightarrow 0
$$

Here $U$ is the Fock space localization $U_{\beta}$ that corresponds to $\beta=\{1, \ldots, N\}$.

Proof. Since the energy of $\Psi_{n}$ is uniformly bounded we also know that

$$
\left(\Psi_{n}, H^{0}(m) \Psi_{n}\right) \leq C
$$

is uniformly bounded 
Let us describe the operator $\mathcal{I} \otimes P_{2} U$ in more detail. Recall that

$$
U a^{*}\left(h_{i_{1}}\right) \cdots a^{*}\left(h_{i_{k}}\right)\left|0>=c^{*}\left(h_{i_{1}}\right) \cdots c^{*}\left(h_{i_{k}}\right) U\right| 0>
$$

where $\mid 0>$ denotes the vacuum vector in Fock space and $U|0>=| 0>\otimes \mid 0>$. Hence, using the definition of $c^{*}(h)$, we find that

$$
\mathcal{I} \otimes P_{2} U a^{*}\left(h_{i_{1}}\right) \cdots a^{*}\left(h_{i_{k}}\right)\left|0>=a^{*}\left(h_{i_{1}} j_{1}\right) \cdots a^{*}\left(h_{i_{k}} j_{1}\right)\right| 0>\otimes \mid 0>.
$$

The projection $P_{2}$ annihilates the photons in the second factor. In other words, the operator $\mathcal{I} \otimes P_{2} U$ when acting on a state

$$
\Psi=\left\{\Psi^{0}, \Psi^{1}\left(y_{1}\right), \Psi^{2}\left(y_{1}, y_{2}\right), \cdots\right\}
$$

produces the localized state $\Gamma\left(j_{1}\right) \Psi \otimes \mid 0>$ where

$$
\Gamma\left(j_{1}\right) \Psi=\left\{\Psi^{0}, j_{1}\left(y_{1}\right) \Psi^{1}\left(y_{1}\right), j_{1}\left(y_{1}\right) j_{1}\left(y_{2}\right) \Psi^{2}\left(y_{1}, y_{2}\right), \cdots\right\} .
$$

It follows that

$$
\begin{aligned}
\left(\phi_{R} \Psi_{n}, U^{*} \mathcal{I} \otimes P_{2} U \phi_{R} \Psi_{n}\right) & =\left\|\mathcal{I} \otimes P_{2} U \phi_{R} \Psi_{n}\right\|^{2} \\
& =\left\|\Gamma\left(j_{1}\right) \phi_{R} \Psi_{n}\right\|^{2}
\end{aligned}
$$

Next, we show that (105) implies that

$$
\Gamma\left(j_{1}\right) \phi_{R} \Psi_{n} \rightarrow 0
$$

To achieve that we note first that on account of the positive mass we have that $\left(\Psi_{n}, \mathcal{N} \Psi_{n}\right)$ is uniformly bounded. Since $\Psi_{n}$ is of the form

$$
\left\{\Psi_{n}^{0}, \Psi_{n}^{1}\left(X, y_{1}\right), \Psi_{n}^{2}\left(X, y_{1}, y_{2}\right), \cdots\right\}
$$

we know that $\sum_{k \geq M}\left(\Psi_{n}^{k}, \Psi_{n}^{k}\right) \leq$ const/M. It is therefore sufficient to prove (110) for each function

$$
\Psi_{n}^{M}\left(X, y_{1}, \cdots, y_{M}\right)
$$

From the lemma below we learn that

$$
\sum_{j=1}\left(\Psi_{n}, p_{j}^{2} \Psi_{n}\right)
$$

is uniformly bounded. Thus, we can write (110) as

$$
\Gamma\left(j_{1}\right) \phi_{R}\left(1+\sum_{j=1} p_{j}^{2}+H_{f}\right)^{-1 / 2}\left(1+\sum_{j=1} p_{j}^{2}+H_{f}\right)^{1 / 2} \Psi_{n}
$$

which vanishes as $n \rightarrow \infty$ since $\left\|\left(1+\sum_{j=1} p_{j}^{2}+H_{f}\right)^{1 / 2} \Psi_{n}\right\|$ is uniformly bounded and since

$$
\Gamma\left(j_{1}\right) \phi_{R}\left(1+\sum_{j=1} p_{j}^{2}+H_{f}\right)^{-1 / 2}
$$

is compact on every finite particle subspace . Compactness follows from the fact that for continuous functions $f$ and $g$ vanishing at infinity the operator $f(i \nabla) g(x)$ is compact. 
A.4. LEMMA (Bound on $\left.A(x)^{2}\right)$. For each $x \in \mathbb{R}^{3}$ and ultraviolet cutoff $\Lambda$ write $A(x)=$ $D(x)+D^{*}(x)$ where $D$ contains the annihilation operators in $A(x)$ and $D^{*}$ the creation operators. Similarly, write $B(x)=E(x)+E^{*}(x)$. As operator bounds

$$
\begin{aligned}
H_{f} & \geq \frac{1}{8 \pi \Lambda} D^{*}(x) D(x) \\
H_{f}+\frac{\Lambda}{2} & \geq \frac{1}{8 \pi \Lambda} D(x) D^{*}(x) \\
H_{f}+\frac{\Lambda}{8} & \geq \frac{1}{32 \pi \Lambda} A(x)^{2} \\
H_{f} & \geq \frac{3}{8 \pi \Lambda^{3}} E^{*}(x) E(x) \\
H_{f}+\frac{3 \Lambda}{4} & \geq \frac{3}{8 \pi \Lambda^{3}} E(x) E^{*}(x) \\
H_{f}+\frac{3 \Lambda}{16} & \geq \frac{3}{32 \pi \Lambda^{3}} B(x)^{2} .
\end{aligned}
$$

Proof. We write $A(x)=D(x)+D^{*}(x)$ with $D(x)=\sum_{\lambda} \int_{|k|<\Lambda} \varepsilon_{\lambda}(k) \exp [i k \cdot x] a_{\lambda}(k) d^{3} k$. There are thus four terms in $A(x)^{2}$. Using the Schwarz inequality, the $(D D)$ term can be bounded above by $\left(D^{*} D\right) / 2+\left(D D^{*}\right) / 2$. On the other hand, $\left(D D^{*}\right)=\left(D^{*} D\right)+\Gamma$, where $\Gamma$ is the commutator $\int 2 /|k|=4 \pi \Lambda^{2}$; the factor 2 comes from the two polarizations $\lambda=1,2$. Altogether, we obtain

$$
A(x)^{2} \leq 4 D^{*}(x) D(x)+4 \pi \Lambda^{2} .
$$

Finally, we use the Schwarz inequality again to obtain

$$
\sum_{\lambda} \int \overline{h_{\lambda}(k)} a_{\lambda}^{*}(k) d^{3} k \sum_{\lambda} \int h_{\lambda}(k) a_{\lambda}(k) d^{3} k \leq \sum_{\lambda} \int\left|h_{\lambda}(k)\right|^{2} /|k| d^{3} k \sum_{\lambda} \int|k| a_{\lambda}^{*}(k) a_{\lambda}(k) d^{3} k .
$$

In our case, $h_{\lambda}(k)=\varepsilon_{\lambda}(k) \exp [i k \cdot x] / \sqrt{|k|}$, so $\sum_{\lambda} \int\left|h_{\lambda}(k)\right|^{2} /|k| d^{3} k=8 \pi \Lambda$.

For $B=\operatorname{curl} A$, replace $\Gamma$ by $2 \pi \Lambda^{4}$ and replace $\left|h_{\lambda}(k)\right|$ by $\sqrt{|k|}$.

As a corollary of Lemma A.4 we have the following.

A.5. LEMMA (Bound on $\left.(p+A(x))^{2}\right)$. For any $\varepsilon>0$ there are constants $\delta(\varepsilon)>0$ and $C(\varepsilon)<$ $\infty$ such that

$$
\sum_{j=1}^{N}\left\{\left(p_{j}+\sqrt{\alpha} A\left(x_{j}\right)\right)^{2}+\frac{g}{2} \sqrt{\alpha} \sigma_{j} \cdot B\left(x_{j}\right)\right\}+\varepsilon H_{f} \geq \delta(\varepsilon) \sum_{j=1}^{N} p_{j}^{2}-C(\varepsilon) .
$$

The constants $\delta(\varepsilon), C(\varepsilon)$ depend on $\alpha, g, \Lambda, N$.

Proof. In addition to Lemma A.4, use the facts that for any $0<\mu, \nu<1,\left(p_{j}+\sqrt{\alpha} A\left(x_{j}\right)\right)^{2} \geq$ $(1-\mu) p^{2}+(1-1 / \mu) \alpha A\left(x_{j}\right)^{2}$ and $2 \sigma_{j} \cdot B\left(x_{j}\right) \geq-\nu B\left(x_{j}\right)^{2}-1 / \nu$.

\section{References}

[1] S. Agmon, Lectures on exponential decay of solutions of second order elliptic equations: Bounds on eigenfunctions of $N$-body Schrödinger operators, Mathematical Notes 29, Princeton University Press, (1982). 
[2] A. Arai, Rigorous theory of spectra and radiation for a model in quantum electrodynamics J. Math. Phys. 24, 1896-1910 (1983).

[3] A. Arai and M. Hirokawa, On the existence and uniqueness of ground states of a generalized spin-boson model J. Funct. Anal. 151, 455-503 (1997).

[4] A. Arai and M. Hirokawa, Ground states of a general class of quantum field Hamiltonians, preprint mp_arc 99-179 (1999).

[5] A. Arai, M. Hirokawa and F. Hiroshima, On the absence of eigenvectors of Hamiltonians in a class of massless quantum field models without infrared cutoff, J. Funct. Anal. (in press).

[6] V. Bach, J. Fröhlich and I.M. Sigal, Mathematical Theory of Nonrelativistic Matter and Radiation, Lett. Math. Phys. 34, 183-201 (1995).

[7] V. Bach, J. Fröhlich and I.M. Sigal, Quantum electrodynamics of confined non-relativistic particles, Adv. Math. 137, 299-395 (1998).

[8] V. Bach, J. Fröhlich and I.M. Sigal, Spectral analysis for systems of atoms and molecules coupled to the quantized radiation field, Commun. Math. Phys. 207, 249-290 (1999).

[9] J. Combes and L. Thomas, Asymptotic behavior of eigenfunctions for multiparticle Schrödinger operators, Commun. Math. Phys. 34, 251-270 (1973).

[10] J. Dereziński and C. Gérard, Asymptotic completeness in quantum field theory. Massive PauliFierz Hamiltonians, Rev. Math. Phys. 11, 383-450 (1999).

[11] M. Fierz and W. Pauli, Nuovo Cim. 15, 167 (1938).

[12] C. Gérard, On the existence of ground states for massless Pauli-Fierz Hamiltonians, preprint mp_arc 99-158 (1999).

[13] M. Hirokawa, Remarks on the Ground state energy of the Spin-Boson model. An application of the Wigner-Weisskopf model. preprint mp_arc 00-239 (2000).

[14] F. Hiroshima, Ground states of a model in nonrelativistic quantum electrodynamics I and II, J. Math. Phys. 40, 6209-6222 (1999), 41, 661-674 (2000).

[15] F. Hiroshima and H. Spohn, Binding through coupling to a field, preprint (1999).

[16] W. Hunziker and I.M. Sigal, The general theory of $N$-body quantum systems, in Mathematical quantum theory. II. Schrödinger operators (Vancouver, BC, 1993), 35-72, CRM Proc. Lecture Notes, 8, Amer. Math. Soc., Providence, RI, 1995.

[17] E.H. Lieb and M. Loss, Self-Energy of Electrons in Non-perturbative QED , in Differential Equations and Mathematical Physics, University of Alabama, Birmingham, 1999, R. Weikard and G. Weinstein, eds. 255-269 Internat. Press (1999). arXiv math-ph/9908020, mp_arc 99-305. 
[18] E.H. Lieb and M. Loss, Analysis, Graduate Studies in Mathematics, American Mathematical Society, 1997.

[19] A. O'Connor, Exponential decay of bound-state wave functions, Commun. Math. Phys. 32, 319-340 (1973).

[20] M. Reed and B. Simon, Methods of modern mathematical physics, vol 4. Theorem XIII.39, Academic Press (1978).

[21] H. Spohn, Asymptotic completeness for Rayleigh scattering, J. Math. Phys. 38, 2281-2296 (1997).

[22] H. Spohn, Ground state(s) of the spin-boson Hamiltonian, Commun. Math. Phys. 123, 277-304 (1989).

[23] H. Spohn, Ground state of a quantum particle coupled to a scalar Bose field, Lett. Math. Phys. 44, 9-16 (1998).

marcel@vorteb.math.uab.edu

lieb@princeton.edu

loss@math.gatech.edu 